\title{
Approche expérimentale de la navigation précolombienne dans les Antilles
}

Experimental approach of the pre-Columbian navigation in the Antilles

Enfoque experimental sobre la navegación precolombina en las Antillas

Benoît Bérard, Jean-Yves Billard, Thierry L'Etang, Guillaume Lalubie, Costantino Nicolizas, Bruno Ramstein et Emma Slayton

\section{(2) OpenEdition}

Journals

Édition électronique

URL : https://journals.openedition.org/jsa/14843

DOI : 10.4000/jsa. 14843

ISSN : 1957-7842

Éditeur

Société des américanistes

Édition imprimée

Date de publication : 31 décembre 2016

Pagination : 171-204

ISSN : 0037-9174

Référence électronique

Benoît Bérard, Jean-Yves Billard, Thierry L'Etang, Guillaume Lalubie, Costantino Nicolizas, Bruno Ramstein et Emma Slayton, « Approche expérimentale de la navigation précolombienne dans les Antilles », Journal de la Société des américanistes [En ligne], 102-2 | 2016, mis en ligne le 26 janvier 2017, consulté le 02 septembre 2022. URL : http://journals.openedition.org/jsa/14843; DOI : https:// doi.org/10.4000/jsa. 14843 
Note DE RECHERCHE 



\title{
Approche expérimentale de la navigation précolombienne dans les Antilles
}

\author{
Benoît Bérard, Jean-Yves Billard, Thierry L'Etang, Guillaume Lalubie, \\ Costantino Nicolizas, Bruno Ramstein et Emma Slayton *
}

\begin{abstract}
Devenue plus centrale au cours des dernières années, la question des capacités de navigation des populations amérindiennes n'est pas nouvelle dans l'archéologie antillaise. Nous avons choisi de principalement concentrer nos efforts sur une approche expérimentale de ces techniques. Notre ambition était de mettre en place un programme d'expérimentation prenant en compte les différents aspects liés à la construction et à l'utilisation des kanawa. Nous avons donc mené une étude ethnoarchéologique de la chaîne opératoire de construction des kanawa mise en place par les artisans kali'ña en Guyane française. Puis, une fois produite, la kanawa « Akayouman » a fait l'objet d'une modélisation numérique 3D qui a servi de support à une analyse hydrostatique. Enfin, le cœur du projet a été constitué par la collecte et le traitement des données issues de notre programme de navigation. [Mots-clés : Antilles, navigation amérindienne, ethnoarchéologie, archéologie expérimentale, sociétés maritimes.]
\end{abstract}

Experimental approach of the pre-Columbian navigation in the Antilles. Understanding the navigation capacities of the Eastern Caribbean pre-Columbian population is crucial for the community working in the Antilles. We have chosen to focus our work on an experimental approach of these techniques. Our ambition was to set up an experimental program including all the aspects associated with the building and the utilization of the kanawa. Thus, we have conducted an ethnoarchaeological study of the kanawa building process followed by the Kali'ña from French Guyana. Then, the "Akayouman » kanawa was made into a 3D model to support a hydrostatic analyse. Finally, the heart of the project was composed of gathering and studying the data collected during our expeditions in the kanawa.

* B. Bérard, université des Antilles et de la Guyane, EA 929 AIHP/GEODE et UMR 8096 ArchAm [benoit.berard@martinique.univ-ag.fr]; J.-Y. Billard, Institut de recherche de l'École navale, Groupe M2EM, EA 3634 [jean-yves.billard@ecole-navale.fr]; T. L'Etang, association Karisko [thierry.letang@cr-guadeloupe.fr]; G. Lalubie, université des Antilles et de la Guyane, EA 929 AIHP/GEODE [g.lalubie@hotmail.fr]; C. Nicolizas, EHESS, LIAS, IMM-UMR 8178 CNRS/EHESS [cobimaltese@gmail.com]; B. Ramstein, association Karisko[ramsteinlb@wanadoo.fr]; E. Slayton, Leiden University, Caribbean Research Group, NWO Island Network [e.r.slayton@arch.leidenuniv.nl]. 
[Key words: Antilles, Amerindian navigation, ethno-archaeology, experimental archaeology, maritime societies.]

Enfoque experimental sobre la navegación precolombina en las Antillas. La comprensión de las capacidades de navegación de las poblaciones precolombinas del Caribe Oriental es actualmente fundamental para la comunidad que trabaja en las Antillas. Hemos optado por centrar nuestro trabajo en una practica experimental de estas técnicas. Nuestra ambición era crear un programa de experimentación que incluye todos los aspectos asociados a la construcción y la utilización de las kanawa. Por lo tanto, hemos realizado un estudio etnoarqueológica del proceso de construcción de las kanawa seguido por los Kali'na de Guyana Francesa. Entonces, la kanawa «Akayouman » ha sido modelizado en 3D para apoyar un estudio hidrostático. Por fin, el corazón del proyecto está constituido por la recopilación y el estudio de los datos recogidos durante nuestras expediciones. [Palabras clave: Antillas, navegación amerindia, etnoarqueología, arqueología experimental, sociedades marítimas.]

À Solange et Marcel, à Gérald, à Akayouman, lonnè épi respé

\section{Introduction}

2 mai 2008 à l'aube, Pointe de Macouba (extrémité nord de l'île de Martinique) Vingt-sept hommes et femmes s'apprêtent à traverser le Canal de la Dominique, bras de mer séparant la Martinique de sa voisine située 23 miles nautiques ( $\left.\mathrm{MN}^{1}\right)$ plus au nord. Pour la première fois depuis trois cents ans, ils vont réaliser cette navigation à la pagaie sur une kanawa, une pirogue monoxyle à fargues de près de dix-huit mètres de long². Cette embarcation se veut conforme à celles utilisées par les Amérindiens des Petites Antilles, les Kalinago (ou Caraïbes insulaires), au moment du contact avec les Européens. Cet équipage pionnier ne sait pas encore que cette première expédition sera suivie par de nombreuses autres.

Entre 2007 et 2010 l'association Karisko a développé un programme d'archéologie expérimentale intitulé Kytangomingo $\mathrm{Ema}^{3}$ et consacré à l'étude des techniques de navigation amérindiennes dans les Antilles (Collectif Karisko 2011). La compréhension des capacités dans ce domaine des populations précolombiennes de la Caraïbe insulaire est aujourd'hui au cœur de nombreuses questions qui

1. Le mille nautique est l'unité de distance utilisée pour la navigation maritime et aérienne, il correspond à $1852 \mathrm{~m}$.

2. Une expédition comparable a été menée par Antonio Nuñez Jiménez en 1987, cependant l'essentiel des traversées a alors été réalisé sous voile avec un canot non conforme aux données historiques auquel un balancier avait été ajouté (Núñez Jiménez 1992 et 1994).

3. Le chemin de nos ancêtres (Kalinago). 
occupent la communauté des archéologues antillanistes (pour une présentation du contexte géographique et historique du peuplement amérindien de l'archipel antillais voir Bérard et al. 2016). Elles concernent principalement: la nature des moyens techniques développés; l'impact des facteurs environnementaux; les possibles connexions entre les îles de l'arc antillais et entre ces dernières et les différentes zones du continent américain; les routes suivies par les différentes migrations à l'origine du peuplement de l'archipel (Fitzpatrick 2013).

Le programme que nous avons mis en place n'avait pas pour vocation première de répondre à l'ensemble de ces questions. Il a pour origine un projet plus vaste dont l'objectif est de développer une géographie sociale des territoires (Di Méo 1998) des premiers groupes agro-céramistes au sein de l'archipel antillais (Bérard 2013a). Or ce type d'approche ne pouvait faire l'économie d'une évaluation sérieuse de la capacité technique de ces populations à développer et à maintenir des relations et des échanges réguliers entre les différentes îles. Très rapidement les études que nous avions menées sur les occupations céramiques anciennes de Martinique (Bérard 2004) et de Dominique (Bérard 2007, 2008 et 2013 b) nous avaient montré qu'il était impératif pour tenter de cerner et de définir ces territoires de quitter une approche terrestre et insulaire et d'adopter une perspective plus maritime et archipélique.

Devenue centrale au cours des dernières années, la question des capacités de navigation des populations amérindiennes n'est cependant pas nouvelle dans l'archéologie antillaise. Elle a été traditionnellement traitée selon deux voies: l'étude des textes européens du moment du contact (McKusick 1960; Nicholson 1976) et le développement à partir de ces éléments et de données environnementales de simulations numériques (Altes 2011; Callaghan 2001, 2011 et 2013). Ces démarches riches d'apports ont cependant été marquées par deux types de limites. Tout d'abord une exploitation trop partielle des sources historiques, principalement centrée sur les premières chroniques espagnoles, laissait un grand nombre de questions ouvertes (morphologie précise des embarcations, capacités de chargement, utilisation précolombienne de la voile...). Ensuite les différentes simulations produites, basées sur la modélisation des phénomènes de dérive, n'intègrent pas, faute de données disponibles, le facteur humain ou la capacité des embarcations à supporter les différents états de la mer. Elles se sont concentrées sur l'évaluation des possibilités de voyages de découvertes. Il paraissait donc nécessaire de produire de nouvelles données afin de mieux répondre à la diversité des questionnements actuels. La mise en place d'un programme d'archéologie maritime expérimentale nous est apparue comme l'un des moyens propres à pallier ce manque.

L'archéologie maritime expérimentale a été depuis plus d'un demi-siècle au cœur de nombreux projets à l'intérêt scientifique parfois discutable (Capelotti 2012). Elle a cependant largement démontré sa capacité à produire 
des données originales essentielles à la compréhension du fait maritime tout au long de l'histoire (Crumlin-Pedersen et Vinner 1986; Bennet 2009). Ces données concernent tant les techniques de construction que l'utilisation des embarcations reproduites sous une forme ou une autre (simulation numérique, maquettes, véritables répliques). Le recours à l'archéologie maritime expérimentale présente cependant un certain nombre de difficultés depuis longtemps identifiées (Christensen et Morisson 1976). Elles concernent principalement la conformité historique du processus de construction et de la morphologie des embarcations ainsi que le difficile contrôle de l'ensemble des données lors des expériences de navigation ${ }^{4}$. Des décennies de pratique ont malgré tout permis de mettre au point un cadre théorique et méthodologique permettant de limiter l'impact de ces difficultés (Coates et al. 1995; Crumlin-Petersen 1995; Crumlin-Petersen et McGrail 2006; McGrail 2009). C’est dans ce cadre général que nous avons souhaité inscrire notre recherche.

\section{Méthodologie}

Tout projet d'archéologie maritime expérimentale est une aventure collective de par la diversité des savoirs mobilisés, la nécessité de doter l'embarcation produite d'un équipage et du fait des contraintes d'intendance et de sécurité inhérentes à ce type de pratique. L'équipe scientifique que nous avons réunie est ainsi constituée d'archéologues, d'historiens, d'anthropologues, de charpentiers de marine, de physiciens et de géographes. Le recrutement et la formation de l'équipage ont été rendus possibles par le dynamisme de l'association Karisko qui a aussi pris en charge la gestion des lourdes questions d'intendance et de sécurité.

Afin d'assurer la plus grande conformité historique de l'embarcation reproduite, la première étape de notre travail fut de faire un inventaire complet des données archéologiques directes (restes d'embarcations et de pagaies) liées à la pratique de la navigation précolombienne dans les Antilles. Face à la pauvreté des données recueillies, il nous est rapidement apparu indispensable de compléter ce travail par une étude historique systématique des sources européennes. Ce travail avait un autre objectif, celui d'assurer autant que possible la profondeur historique des savoir-faire techniques traditionnels toujours vivants au sein de certaines populations de l'espace antillo-guyanais. Ce sont en effet ces savoirfaire qui nous ont servis pour compléter les éléments laissés dans l'ombre par les

4. Un dernier facteur à prendre en compte est la diversité des objectifs souvent liés à ce type de projet. Comme bien souvent en archéologie maritime expérimentale le projet « Kytangomingo Ema » intégrait des aspects culturels, sociaux et éducatifs. Nous ne les évoquerons ici que lorsqu'ils nous semblent avoir interféré de façon notable avec les objectifs scientifiques du projet. 
données archéologiques et l'étude historique et nous ont permis de produire une embarcation apte à répondre aux exigences de notre étude (Bérard et al. 2016).

La seconde étape du projet a été la production de l'embarcation; pour cela

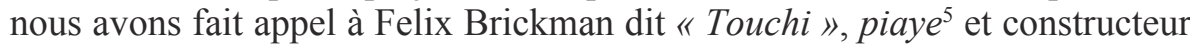
de kanawa kali' ña originaire du Surinam et vivant en Guyane française. Cette étape importante du projet ne sera pas évoquée ici et fera l'objet d'une publication ultérieure. Ensuite 1'embarcation produite a servi de référence pour la réalisation d'un modèle numérique utilisé dans le cadre une étude hydrostatique menée au sein de l'Institut de recherche de l'École navale (Billard et al. 2009). L'intérêt principal de l'étude hydrostatique était de permettre l'élimination de solutions conduisant à des états de chargement du bateau peu probables, le bateau ayant alors une probabilité de chavirement trop élevée. L'étude consista donc, à partir des données objectives concernant la géométrie, le nombre de passagers-pagayeurs, la position de l'équipage lui permettant de ramer de façon efficace, ainsi que la valeur et la position de ces masses indispensables au bon fonctionnement de l'embarcation, à déterminer l'état de stabilité du bateau sans fret, puis de charger progressivement l'embarcation pour déterminer les déplacements et les positions du centre de gravité entre lesquels la stabilité devenait suffisante tout en garantissant un franc-bord minimum compatible avec une navigation maritime. Il a ainsi été montré que les kanawa étaient caractérisées par une forte tendance rouleuse. Cette observation trouve de nombreux échos dans les sources historiques. Ainsi, au-delà des nombreux récits de chavirage que l'adoption de la voile n'a fait que multiplier, le dictionnaire du père Breton nous donne par exemple: « moulíneti boucoúni/le canot roule » (Breton 1665, p. 363). La question du lest est donc essentielle. En effet le navire « serait volage et tournerait sens dessus dessous, pour peu qu'il ne fût pas assez lesté » (Labat 1742 [1694-1705], p. 180). Les résultats de cette étude nous montrent que l'embarcation seule n'aurait pas permis une navigation dans des conditions suffisantes de sécurité pour l'équipage. Il est donc nécessaire de lester la kanawa avec un minimum de $500 \mathrm{~kg}$ de lest ou de fret pour assurer une navigation dans de bonnes conditions, un fret de $1500 \mathrm{~kg}$ semblant être la limite maximum permettant de conserver un franc-bord et un angle de gîte avant entrée d'eau suffisants. Ces données essentielles sur la capacité de chargement de ce type d'embarcations sont une première information importante apte à améliorer notre compréhension des capacités d'échanges interinsulaires des populations précolombiennes des Antilles. Elles nous ont aussi guidés pour la mise en place de notre programme de navigation expérimentale dont la présentation et les apports constituent le cœur du présent article.

5. Terme kali'ña désignant les chamanes (Chalifoux 1998). 


\section{Akayouman, l'esprit du grand-père serpent}

La première étape de la partie expérimentale de notre projet a été le lancement au second semestre 2007 du chantier de construction de deux kanawa. Pour cela nous avons choisi de nous appuyer sur le savoir-faire de groupes amérindiens contemporains. Un premier contact a ainsi été pris avec les Kalinago de la Dominique dont certains continuent à produire régulièrement des gommiers (Figure 1, ci-contre). Malheureusement, il ne leur a pas été possible de répondre favorablement à notre demande faute d'avoir accès à des arbres suffisamment hauts pour pouvoir servir à la production des embarcations de plus de 50 pieds que nous recherchions (les gommiers actuels font généralement 20-25 pieds de long). C'est pour cela que nous nous sommes tournés vers les Kali'ña de la côte des Guyanes qui continuent à produire des pirogues monoxyles à fargues aujourd'hui utilisées, le plus souvent, dans le cadre de navigations côtières et propulsées par un moteur (François 2003). C'est la mise en regard du cahier des charges - fruit de l'étude des sources historiques et archéologiques - et du savoir-faire d'un maître charpentier de marine kali'ña qui a permis d'aboutir à la production de la kanawa que nous avons utilisée pour notre projet. $\mathrm{La}$ faiblesse des données archéologiques et la persistance d'un certain nombre de zones d'ombre suite à l'analyse des sources historiques imposaient ce recours aux compétences d'un charpentier de marine dépositaire de la tradition de construction des pirogues monoxyles à fargues. Ce type de pratique n'a rien d'original et constitue depuis longtemps un des éléments récurrents des programmes d'archéologie maritime expérimentale (McGrail 1974). Cela fait cependant de notre embarcation une « hypothèse flottante » qu'il est important de décrire en détail et dont il faut évaluer les éléments fondant son caractère hypothétique et leur éventuel impact sur les données recueillies.

La kanawa de 54 pieds français (17,52 m), nommée Akayouman (l'esprit du grand-père serpent), est constituée d'une coque taillée dans un tronc d'arbre unique (coque monoxyle) surmontée de deux fargues et fermée, à l'arrière, par un tableau épais. Des membrures et des bancs renforcent le tout (Figure 2, page suivante). Elle a entièrement été réalisée en grignon rouge ${ }^{6}$.

Les relevés effectués en 2008 sur la coque d'Akayouman (Figure 3, pages suivantes) montrent que la partie monoxyle suit la forme du tronc d'origine: elle présente un bulbe d'évasement maximum vers $3 \mathrm{~m}$ à partir de l'avant. La structure est renforcée par 13 membrures, espacées de 1 à $1,2 \mathrm{~m}$, par une fausse étrave liant les fargues à l'avant et un tableau arrière massif de $8 \mathrm{~cm}$ d'épaisseur comportant les encoches de maintien de la pagalle, grande pagaie qui sert à

6. Ocotea rubra. 


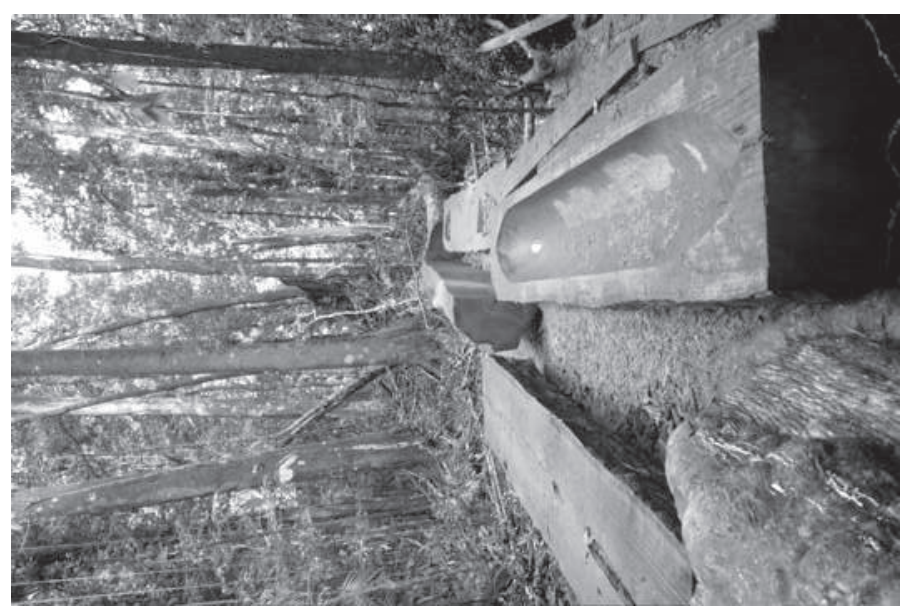

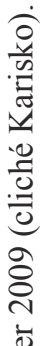

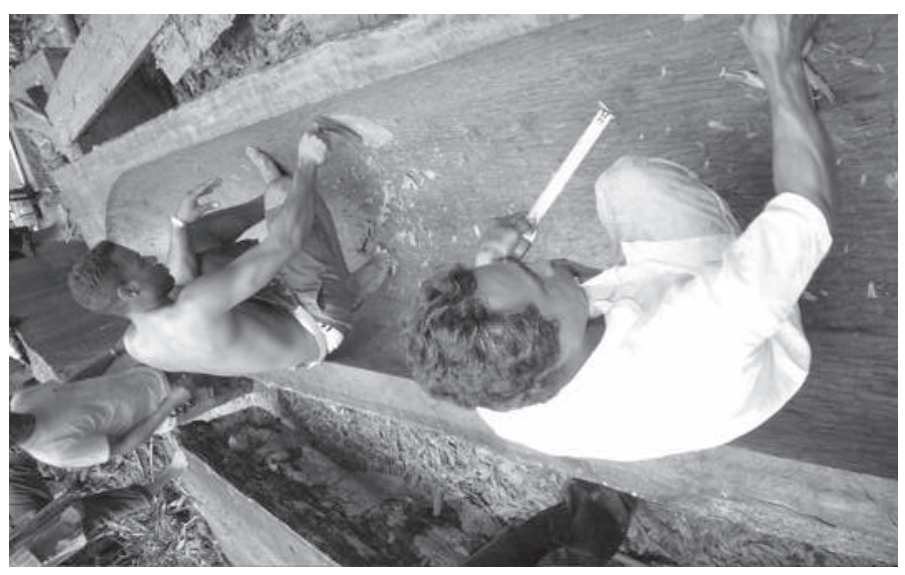

胥

。

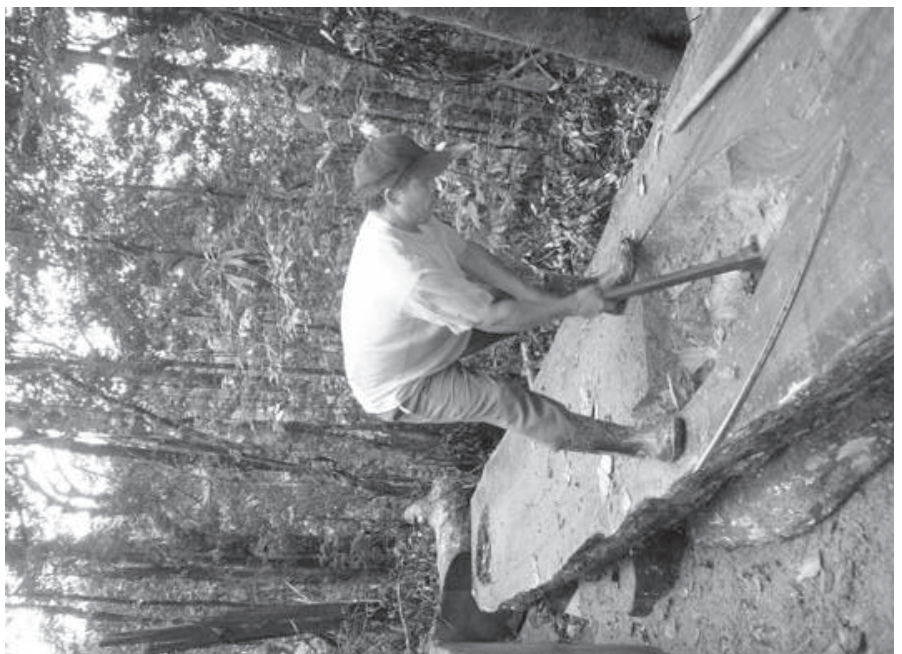




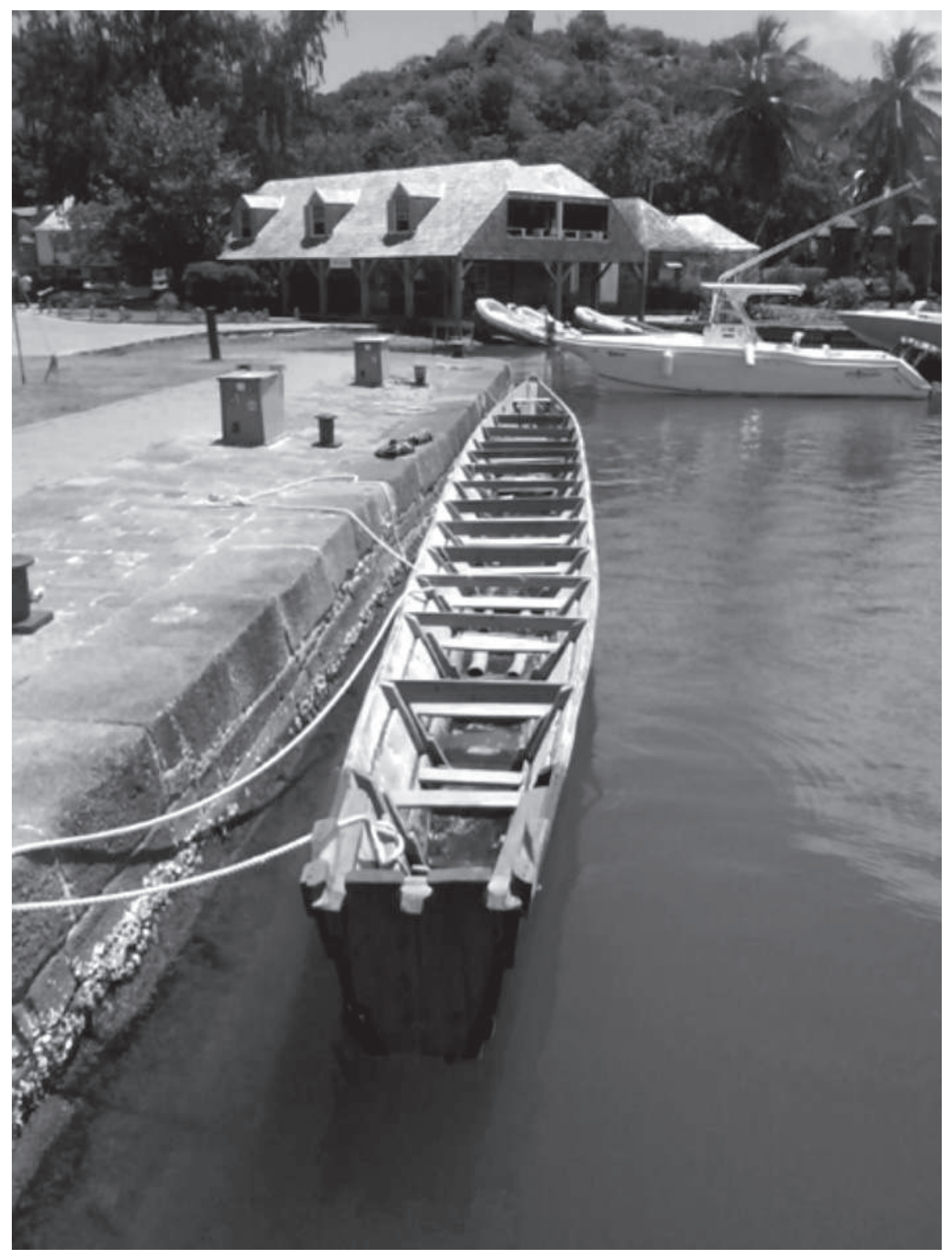

Fig. 2 - Accostée aux Nelson's Dockyard (English Harbour, Antigua), Akayouman se repose de sa longue traversée depuis la Martinique, mai 2009 (cliché Karisko). 


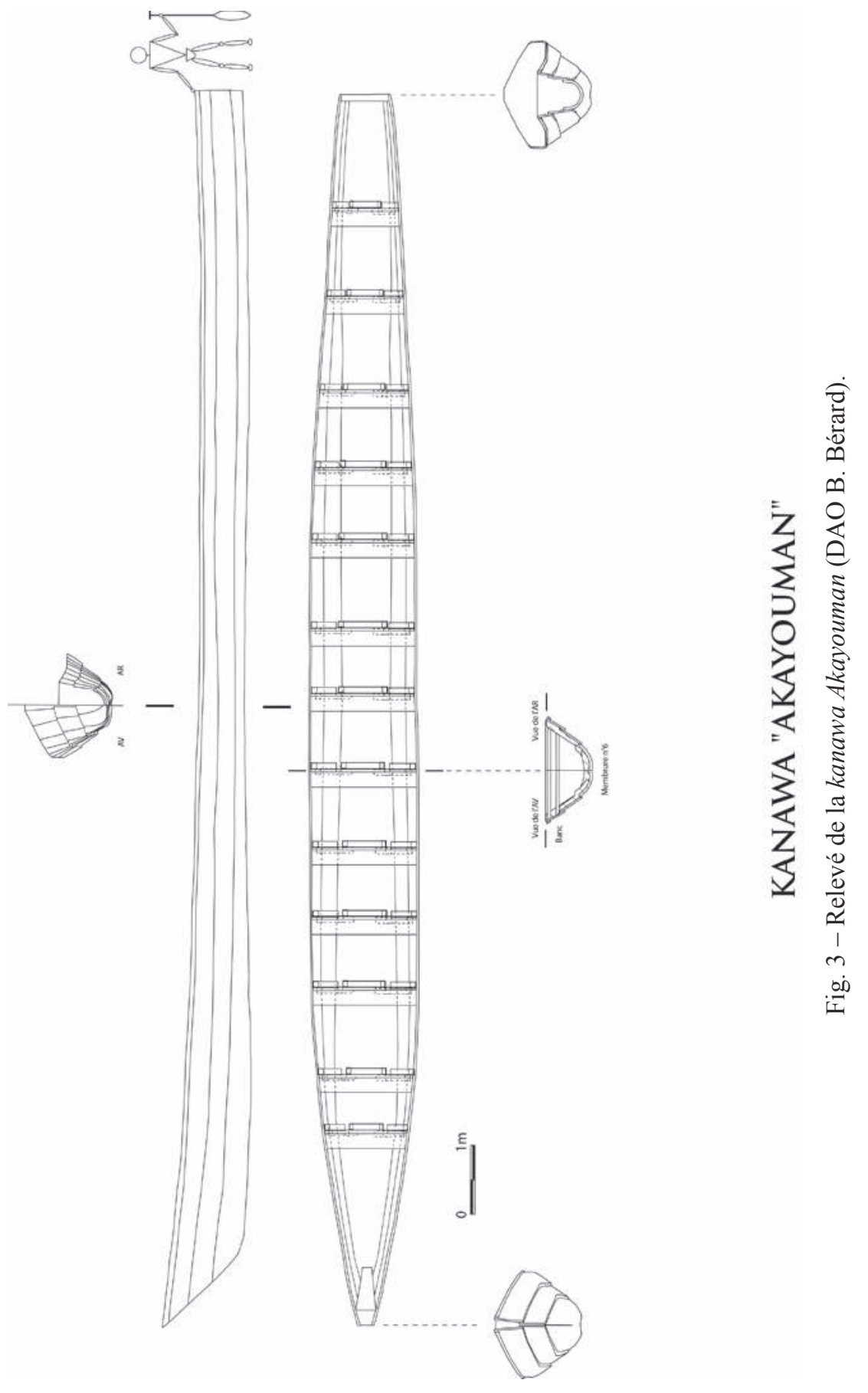




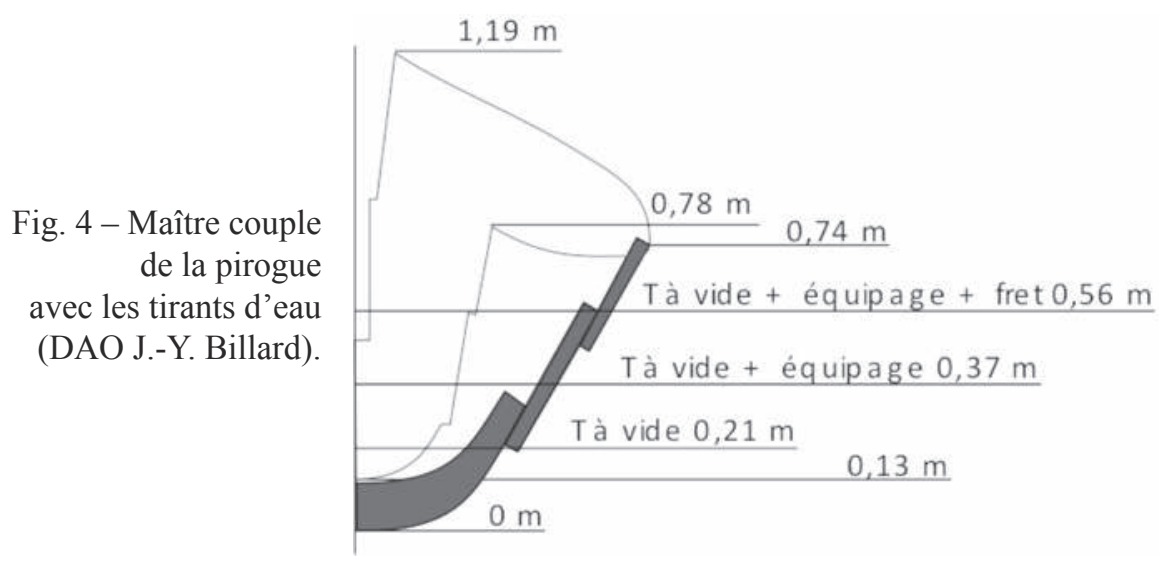

gouverner ${ }^{7}$. Chaque membrure comporte une varangue ( $6 \mathrm{~cm}$ au droit), pourvue d'anguillers, des allonges ( $5 \mathrm{~cm}$ au droit) pour tenir les fargues, un banc à l'avant et un cale-pied, ainsi qu'une traverse dossier à l'arrière. L'épaisseur résiduelle $\mathrm{du}$ « bois fouillé », mis à part le massif avant, varie entre $8 \mathrm{~cm}$ au niveau du bulbe et $5 \mathrm{~cm}$. Celle des fargues est de 2,5 cm. Des vis et des pointes disposées en respectant, autant que possible, les anciennes lignes supposées de couture en fibres végétales assurent les liaisons. Le calfatage est réalisé avec des fibres végétales tropicales et du bitume.

Les dimensions de la kanawa Akayouman sont: longueur: 17,52 m; largeur: $1,5 \mathrm{~m}$; hauteur minimum de bordé : $0,74 \mathrm{~m}$.

À vide elle pèse $1,4 \mathrm{t}$ et son déplacement maximum est de $8 \mathrm{t}$, ce qui laisse de larges possibilités d'emploi : pirogue de guerre embarquant de nombreux guerriers ou bateau de charge adapté au peuplement et aux échanges. En observant le maître couple du bateau (Figure 4, ci-dessus), on remarque, en particulier en partie basse, le tronc évidé qui constitue l'ensemble quille-fonds et qui est particulièrement massif. On a également reporté sur ce vertical la ligne d'étrave, le tableau arrière et le livet donnant ainsi l'extension verticale de la pirogue, ainsi que les tirants d'eau à vide, lège et en charge. À partir des dimensions du bateau et de la position que doit avoir un équipier pour pouvoir pagayer dans de bonnes conditions, on peut estimer le nombre d'équipiers et leur position par rapport au bateau. Ici, 26 pagayeurs, un barreur et un maître d'équipage peuvent prendre place à bord ce qui conduit à un équipage (moyenne de $75 \mathrm{~kg}$ par équipier) pesant environ 2,1 t. Ceci donne, pour l'état lège (pirogue armée) : déplacement: $3,5 \mathrm{t}$; position du centre de gravité : $8,5 \mathrm{~m}$ à partir du tableau et $0,49 \mathrm{~m}$ au-dessus du point le plus bas.

7. Cette morphologie a été inspirée par celle des tableaux arrières des yoles rondes de Martinique qui sont elles aussi dirigées à l'aide d'une pagalle. 
Suite à cette description de la kanawa Akayouman, il est important de repérer les éléments qui paraissent non conformes aux informations historiques et archéologiques (Bérard et al. 2016) et, en fonction des objectifs du projet, d'en évaluer l'impact possible sur la valeur des données recueillies. L'utilisation d'un outillage et de certains matériaux modernes (tronçonneuse, hache et herminette en métal, clous et bitume) a facilité grandement le travail des charpentiers et a été sans conséquence notable sur la morphologie de l'embarcation. Tout au plus le remplacement d'un latex végétal par du bitume en a-t-il renforcé l'étanchéité. La principale différence se situe, en fait, à la poupe: l'utilisation du moteur hors-bords sur les embarcations contemporaines nécessite l'installation d'un tableau arrière relativement large. Akayouman présente cette caractéristique conforme à la pratique actuelle des charpentiers de marine kali'ña. Cependant cette modification localisée en arrière du point le plus large de la pirogue n'influe que peu sur sa progression. Cette largeur de la poupe s'est de fait plutôt révélée un handicap. Lors des navigations réalisées avec une houle venant par l'arrière, les vagues frappaient ce tableau trop large pour les fendre et de l'eau entrait dans l'embarcation. En dehors de ce désagrément, les rares éléments distinguant Akayouman des descriptions historiques semblent donc sans influence notable au regard des données recueillies.

Pour les pagaies, nous nous sommes directement appuyés sur les données archéologiques. Nous avons réalisé des copies de la pagaie Mores découverte aux Bahamas (Booy 1913) et datée de 1436-1511 apr. J.-C. (Ostapkowicz et al. 2012). Cependant une usure de la pointe était évoquée par Booy et plusieurs autres pièces archéologiques antillaises possédaient un rapport pelle/ manche supérieur à la pagaie Mores (Bérard et al. 2016). Nous avons donc décidé d'allonger légèrement la pelle (surface $710 \mathrm{~cm}^{2}$ ) pour constituer notre modèle (Figure 5, page suivante). Une fois la morphologie générale et les rapports de proportion de ce modèle établis, nous l'avons décliné en différentes tailles selon la morphologie des pagayeurs participant au projet dans le respect des rapports de proportions. Le bois devant être souple, nerveux, de droit fil et de contact agréable, nous avons retenu le poirier pays ${ }^{8}$. La grande pagalle (Figure 6, page suivante) qui sert à gouverner a fait l'objet d'améliorations avec l'expérience (longueur totale : 2,40 m; manche: 1,20 m; plat: 1,20 m; largeur: 0,17 m). Le pagayeur d'avant disposait aussi d'une pagalle plus petite, pour aider à la manœuvre.

\section{Pratique expérimentale et données}

La pratique de la navigation en haute mer sur de grandes pirogues monoxyles à fargues s'est depuis longtemps perdue dans les Antilles. Une fois les kanawa

\section{Tabebuia heterophylla.}



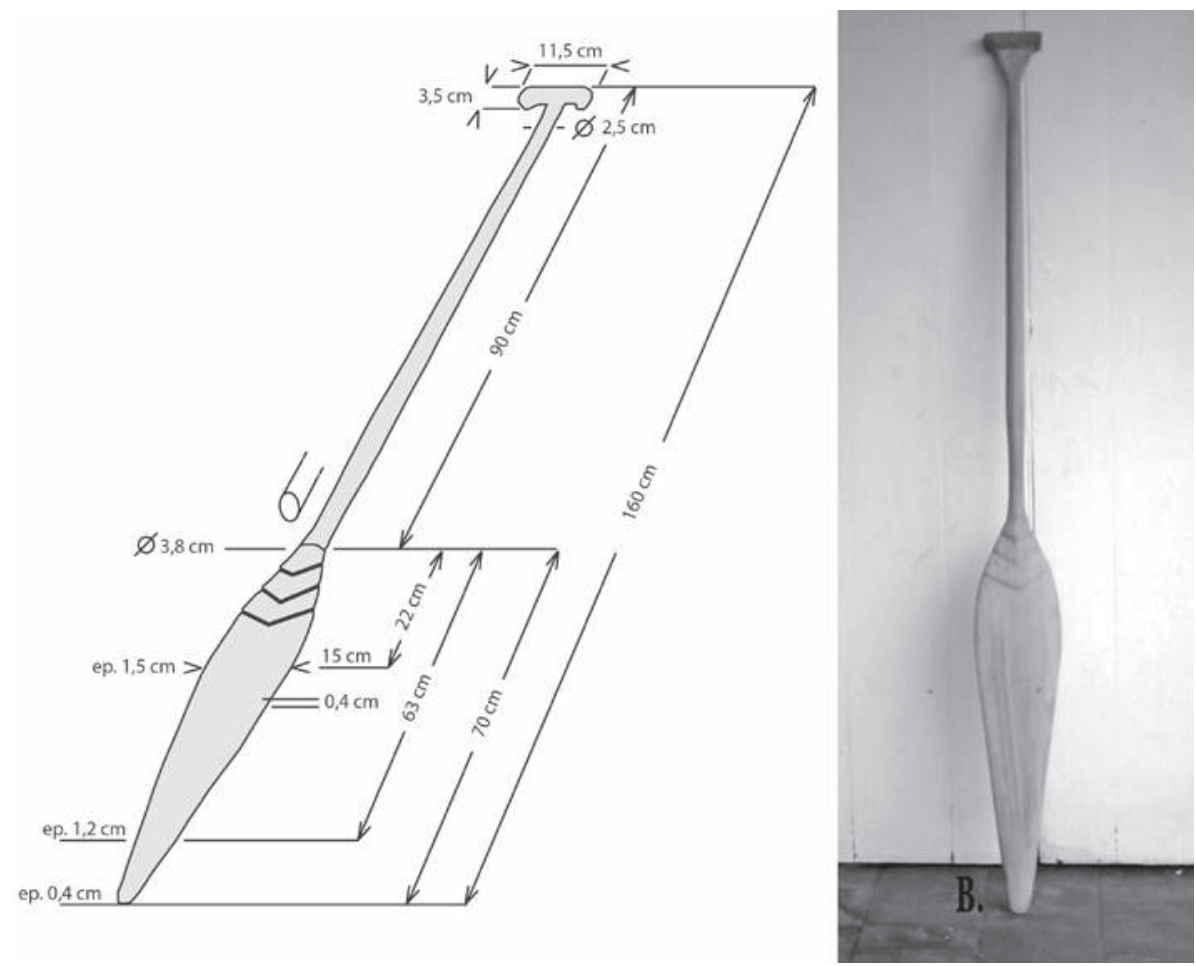

Fig. 5 - Pagaie type utilisée pour le projet déduite de la pagaie de l'île Mores (dessin B. Ramstein, cliché et DAO B. Bérard).

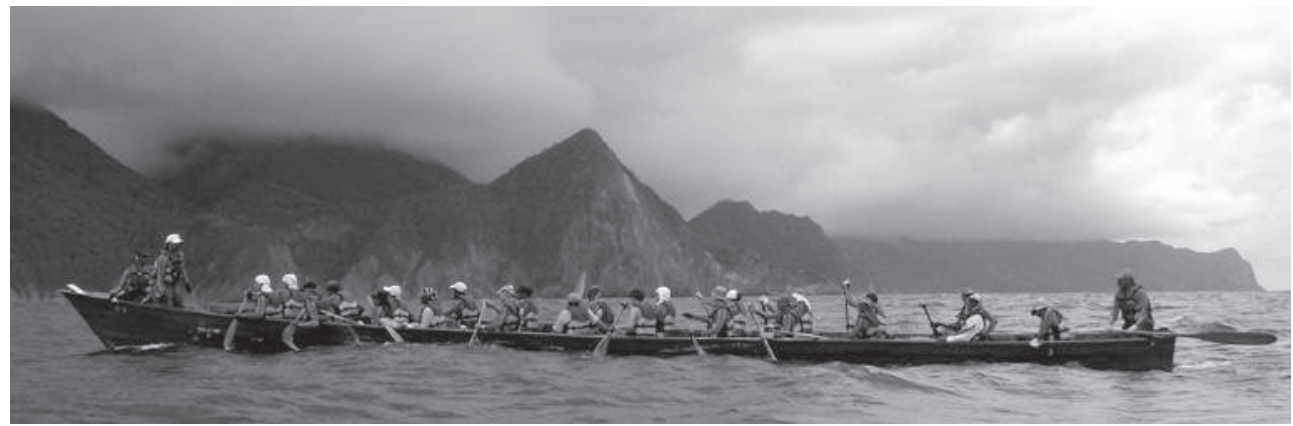

Fig. 6 - Vue de la grande pagalle (cliché Karisko). 
livrées en Martinique, il s'est agi pour nous de composer un équipage et d'entamer un complexe processus de reconstitution des techniques de navigation spécifiques à ce type d'embarcation. Au terme de ce processus nous avons établi un programme de navigation et une procédure adaptée de prise de données qualitatives et quantitatives. Ce dernier point est un sujet d'insatisfaction et une source de débats récurrents lorsqu'il s'agit de discuter de l'apport de tout projet d'archéologie maritime expérimentale. En effet la pratique de la navigation en mer sur des embarcations traditionnelles avec un équipage nombreux est très éloignée de ce que l'on peut considérer comme un environnement contrôlé. Il est difficilement envisageable de rendre compte de l'ensemble des facteurs environnementaux, techniques et humains entrant en jeux. La procédure que nous avons adoptée était donc centrée sur les principaux objectifs qui étaient les nôtres : évaluer les capacités de navigation des kanawa dans des conditions de mer variées en essayant de nous approcher de la définition de conditions limites; produire des données expérimentales solides sur la vitesse de déplacement de ces embarcations; évaluer les capacités d'un équipage à effectuer avec efficacité un effort de pagayage prolongé.

L'enjeu était de produire des données propres à permettre une meilleure évaluation de la durée et de la fréquence possible des déplacements interinsulaires au sein de l'archipel. Il s'agissait par ailleurs d'être apte à discuter sur de nouvelles bases des différentes possibilités de contact entre le continent et les îles ayant été évoquées au cours des dernières années.

\section{L'équipage}

L'équipage standard d'Akayouman se compose de 28 personnes : 26 pagayeurs, 1 maître d'équipage en charge du rythme à l'avant et 1 barreur sur l'arrière. Un des pagayeurs d'avant aide ponctuellement (mer difficile, manœuvres d'accostage) à la direction en jouant le rôle de «propulseur d'étrave ». Entre décembre 2007 et mai 2011, période durant laquelle s'est étendue la prise de données quantitatives ${ }^{9}$, les personnes composant cet équipage ont évidemment changé mais nous avons pu bénéficier d'un petit noyau stable de pratiquants. Le principe général de composition de l'équipage n'a lui pas évolué. En effet l'objectif de notre programme était en priorité d'évaluer les capacités de navigation des populations précolombiennes antillaises dans le cadre de phénomènes migratoires ou de voyages à vocation économique et/ou sociale. Ainsi notre équipage a toujours compris des hommes et des femmes dont l'âge allait de

9. La prise de données qualitatives ne s'est jamais achevée, la pratique de la kanawa se poursuivant jusqu'à ce jour au sein de l'association Karisko. Elle s'est même étendue à l'archipel de la Guadeloupe au travers de l'action de l'association K'nawa a qui nous avons cédé une des deux embarcations construites en Guyane. 
dix-huit à plus de soixante ans ${ }^{10}$. Ces personnes avec ou sans passé sportif avaient dans la plupart des cas une expérience extrêmement faible voire nulle de la navigation. Cela n'a pas posé de problème majeur car les particularités de la navigation à la pagaie en kanawa imposent un apprentissage et une préparation physique spécifiques même pour des personnes ayant une expérience maritime et/ou sportive. Cependant, avant de mettre en place ces procédures d'apprentissage il nous a fallu reconstituer ce savoir-faire.

\section{La reconstitution des savoirs liés à la navigation en kanawa}

Cette reconstitution s'est avant tout basée sur une mosaïque de savoirs ancestraux partiels répartis entre les différents participants au projet. Ils ont été complétés par les fruits de l'expérience progressivement acquise durant le programme. Ainsi, les Kali'ña qui ont réalisé les kanawa avaient une très bonne connaissance des techniques de pagayage mais pas de la haute mer. À l'inverse, les Kalinago de la Dominique qui ont participé au projet avaient l'expérience de la navigation en gommier dans les canaux des Antilles mais au moteur et non à la pagaie. Par ailleurs la pratique extrêmement populaire en Martinique des courses à la voile de gommiers et de yoles rondes fait que la direction de ce type d'embarcation à l'aide d'une pagalle et non d'un gouvernail est quelque chose de connu. Il en est de même des procédures permettant de redresser et de vider ces navires suite à un chavirage. Enfin notre apprentissage a été complété grâce à l'aide de Ronny Matehau. Ce militaire d'origine polynésienne alors en poste en Martinique est moniteur de va'a (pirogue à balancier) et a participé plusieurs fois à la Hawaiki nui va'a, une course internationale reliant les îles de Huahine, Raiatea, Tahaa et Bora-Bora. Son apport a été essentiel quand il s'est agi de passer de la technique individuelle au travail collectif. Cette redécouverte initiale de la navigation en kanawa s'est étendue sur six mois, de décembre 2007 à mai 2008, au rythme de deux entraînements hebdomadaires chacun durant plusieurs heures. Le corpus de connaissances constitué à cette occasion a depuis peu évolué malgré plusieurs années de pratique supplémentaires. Les éléments qui ont demandé un apprentissage plus long sont ceux liés aux interactions complexes avec l'élément maritime permettant la gestion des différents types de conditions de mer (courant, houle, vent, marée) ou la détermination du cap le plus adéquat en fonction de ces conditions. Ils concernent donc plus le maître d'équipage et le barreur que les simples pagayeurs.

10. Nous n'avons dérogé à ce principe qu'à une seule occasion lors d'une traversée Martinique-Dominique en mai 2011, voir ci-après. 


\section{Le programme d'entraînement}

Le programme de navigation que nous avons mis en place entre décembre 2007 et mai 2010 s'est organisé selon une périodicité récurrente. La période allant de novembre à avril était ainsi réservée aux entraînements et à la préparation des expéditions.

Le programme d'entraînement a été conçu et supervisé par des professionnels du sport. Il comprenait de la préparation physique générale (essentiellement de la musculation), du travail technique spécifique (geste de pagayage, synchronisation et maîtrise des différents rythmes) et des sorties longues en mer. Elles se déroulaient le long de la côte sous le vent de la Martinique, leur durée allant croissant pour atteindre 5 à 7 heures à quelques semaines du départ des expéditions. Ce programme intégrait aussi une navigation sur deux jours consécutifs, pour une durée totale entre 12 heures et 14 heures, ainsi que quelques sorties en haute mer. Chaque programme d'entraînement a inclus au moins 100 heures de navigation en kanawa. À cela il faut ajouter différents exercices de sécurité en particulier ceux concernant la procédure permettant de retourner et de vider la kanawa à la suite d'un chavirage afin de pouvoir repartir.

\section{Les expéditions}

Quatre expéditions entre 2008 et 2011 ont servi de support à la prise de données quantitatives. Elles se sont systématiquement déroulées durant le mois de mai. Ce choix a été guidé par notre désir d'éviter, pour des raisons évidentes de sécurité, les épisodes de grandes houles de nord qui touchent régulièrement l'arc antillais entre fin novembre et fin avril ainsi que la saison cyclonique (juillet/ novembre). Il nous permettait aussi de bénéficier des conditions de courant les plus favorables à des navigations dans le sens sud/nord ${ }^{11}$.

Notre première expédition s'est déroulée le 2 mai 2008 et a consisté en une simple traversée du Canal de la Dominique entre la Pointe de Macouba en Martinique et Cachacrou en Dominique (Figures 7 et 8, page suivante). Cette navigation longue d'un peu plus de $24 \mathrm{MN}$ a été réalisée dans des conditions de mer exceptionnellement calmes. Elle a duré 6 h 48 .

La seconde expédition nous a conduits de la Pointe de Macouba, extrémité nord de la Martinique à English Harbour dans le sud d'Antigua (Figure 9 et 10, pages suivantes). Elle s'est déroulée du 21 au 29 mai 2009. Le trajet a été effectué en sept jours de navigation auxquels il faut ajouter deux jours de repos à Portsmouth (nord de la Dominique) et Port-Louis (nord de la Basse-Terre

11. À ce sujet il faut cependant noter que les sources historiques situent l'essentiel des attaques des Caraïbes des Petites Antilles sur Porto Rico en octobre et en novembre. Cette période est marquée pour les Amérindiens par la présence dans le ciel matutinal de la constellation du canot du héron crabier/Loucounni Yabura (Robiou Lamarche 2009, p. 182-192). 
Fig. 7 - Trajet de la première expédition, mai 2008 (DAO B. Bérard).
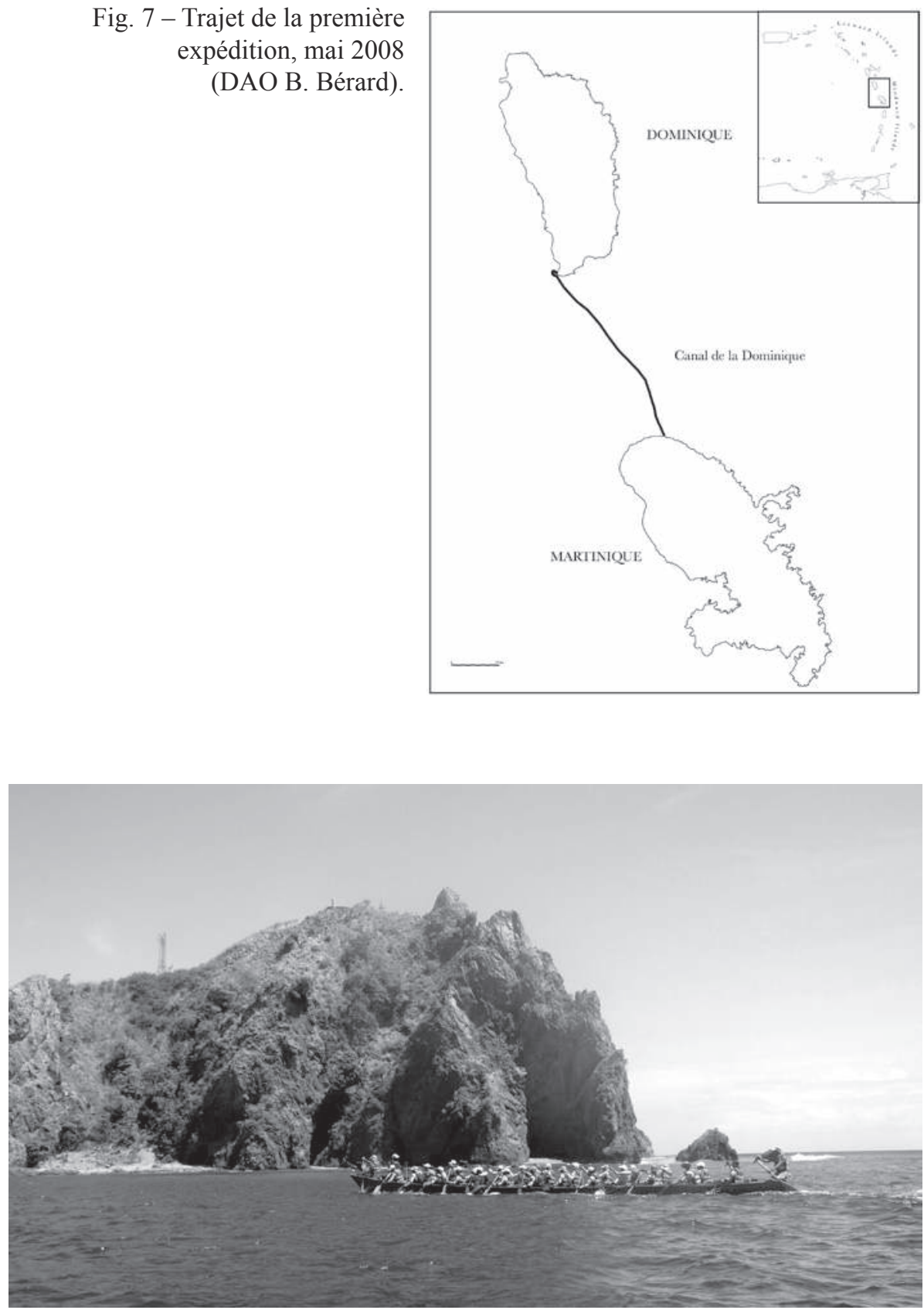

Fig. 8 - Arrivée à Cachacrou (Dominique), 2 mai 2008 (cliché Karisko). 


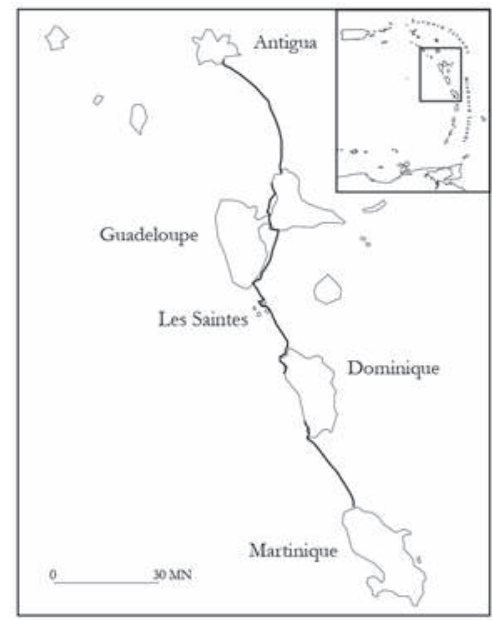

Fig. 9 - Trajet de la deuxième expédition, mai 2009

(DAO B. Bérard).

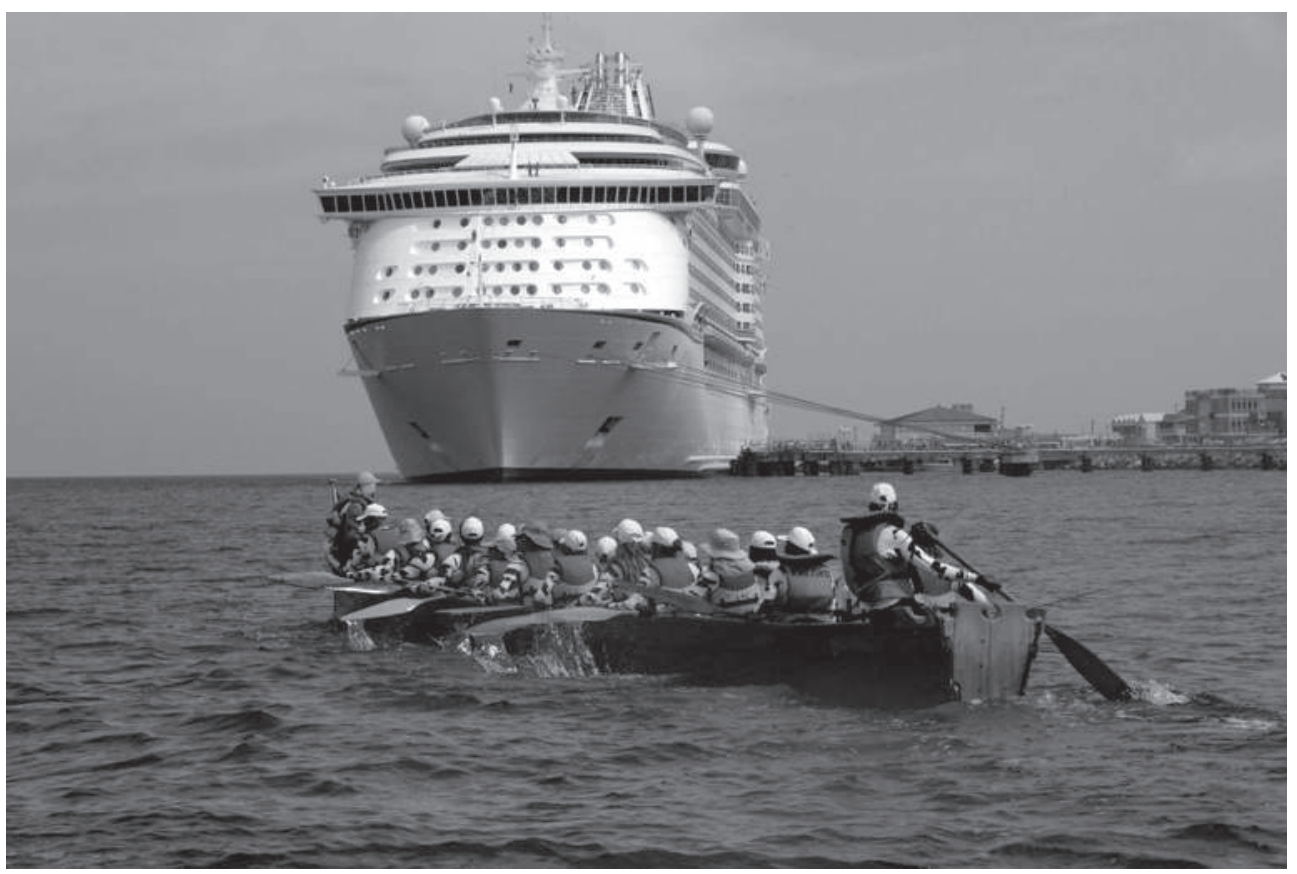

Fig. 10 - La rencontre des deux mondes, Dominique, mai 2009 (cliché Karisko). 
de Guadeloupe). La distance de $139 \mathrm{MN}$ a ainsi été couverte en $43 \mathrm{~h} 55$ de navigation (Figure 11, ci-dessous).

La troisième expédition s'est déroulée du 12 au 22 mai 2010. Elle nous a permis de naviguer dans le sud de l'archipel entre Grenade et Martinique. Cependant, du fait de conditions de mer difficiles et d'une priorité donnée aux aspects culturels, sociaux et éducatifs du programme, la kanawa a été remorquée sur une partie du trajet (Figure 12, ci-contre).

\begin{tabular}{|c|c|c|c|}
\hline Trajet & Date & $\begin{array}{l}\text { Distance } \\
\text { (en MN) }\end{array}$ & Durée \\
\hline $\begin{array}{l}\text { Martinique- } \\
\text { Dominique }\end{array}$ & 21 mai 2008 & 24,6 & $6 \mathrm{~h} 56$ \\
\hline $\begin{array}{l}\text { Sud Dominique- } \\
\text { Nord Dominique }^{\text {a }}\end{array}$ & 22 mai 2008 & 10,5 & $3 \mathrm{~h} 10$ \\
\hline $\begin{array}{c}\text { Nord Dominique- } \\
\text { Les Saintes (Terre } \\
\text { de Haut) }\end{array}$ & 24 mai 2008 & 21,6 & $6 \mathrm{~h} 35$ \\
\hline $\begin{array}{c}\text { Les Saintes-Trois } \\
\text { Rivières (Basse- } \\
\text { Terre, Guadeloupe) }\end{array}$ & 25 mai 2008 & 7,3 & $2 \mathrm{~h} 29$ \\
\hline $\begin{array}{c}\text { Basse-Terre (Trois } \\
\text { Rivière)-Grande } \\
\text { Terre (Gosier) }\end{array}$ & 26 mai 2008 & 19,7 & $8 \mathrm{~h} 25$ \\
\hline Gosier-Port Louis & 27 mai 2008 & 17,9 & $5 \mathrm{~h} 20$ \\
\hline $\begin{array}{l}\text { Pointe de la Grande } \\
\text { Vigie-English } \\
\text { Harbour (Antigua) }\end{array}$ & 29 mai 2008 & 37,3 & $11 \mathrm{~h} 35$ \\
\hline TOTAL & & 139 & $43 \mathrm{~h} 55$ \\
\hline
\end{tabular}

Fig. 11 - Données générales de l'expédition Martinique-Antigua, mai 2009.

Ainsi sur les $170 \mathrm{MN}$ du trajet, seuls 82,3 MN ont été réalisés en kanawa pour une durée totale de pagayage de 31 h 37 réparties sur huit journées (Figure 13, pages suivantes). Toute la partie sud du trajet Grenade-Saint-Vincent a été effectuée dans des conditions difficiles (fort courant, houle, vent et pluie) et changeantes (Figure 14, ci-contre). Ce dernier point est une des spécificités de la navigation au sein des Grenadines.

12. La kanawa a été remorquée sur une partie de ce parcours réalisé sous le vent de la Dominique et ne présentant que peu d'intérêt au regard des centaines d'heures de navigation déjà réalisées dans ce type de conditions. 


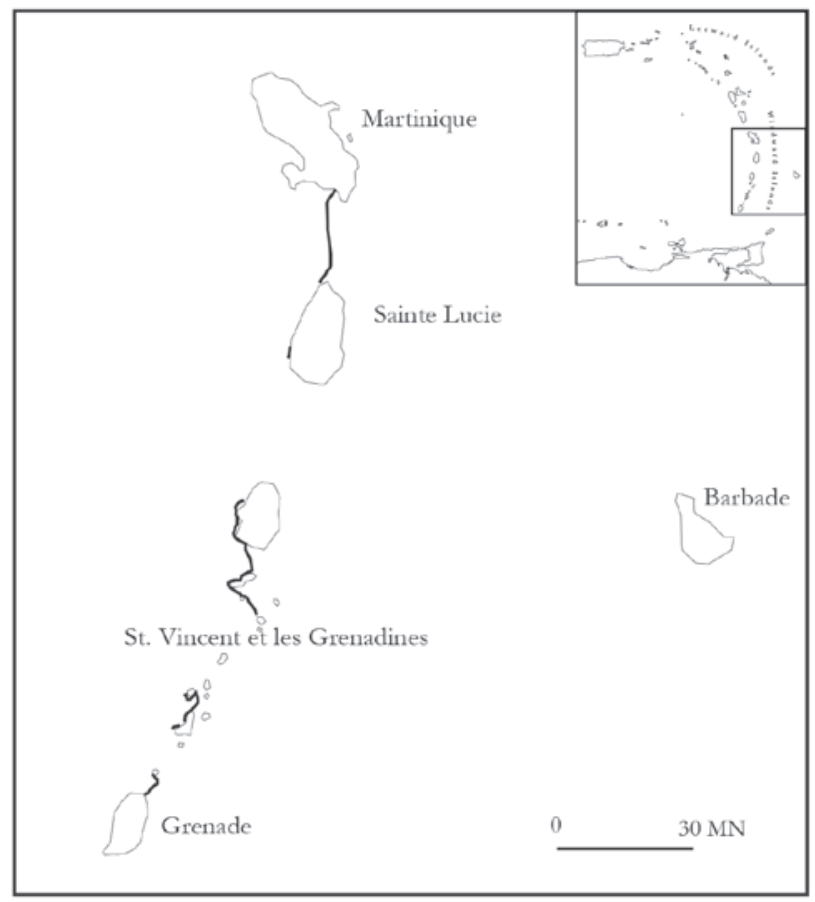

Fig. 12 - Trajet de la troisième expédition, mai 2010 (DAO B. Bérard).

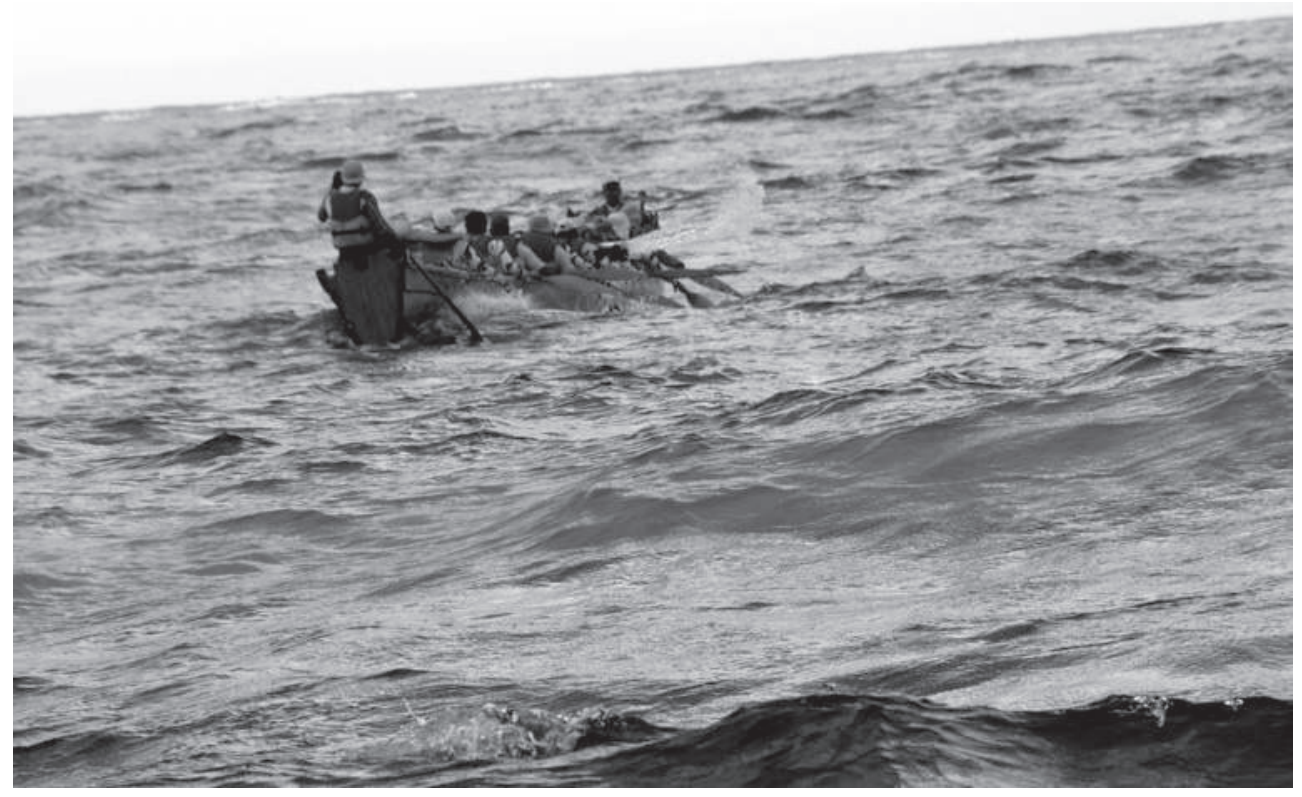

Fig. 14 - Navigation en mer formée entre Grenade et Cariacou, mai 2010 (cliché Karisko). 


\begin{tabular}{|c|c|c|c|}
\hline Trajet & Date & $\begin{array}{c}\text { Distance } \\
\text { (en MN) }\end{array}$ & Durée \\
\hline Grenade-Île Ronde & 12 mai 2010 & 7,6 & $5 \mathrm{~h} 57$ \\
\hline Cariacou-Cariacou & 12 mai 2010 & 1,6 & $0 \mathrm{~h} 38$ \\
\hline Carriacou-Union & 13 mai 2010 & 8 & $1 \mathrm{~h} 32$ \\
\hline $\begin{array}{c}\text { Clifton-Chatham } \\
\text { (Union) }\end{array}$ & 15 mai 2010 & 4,6 & $4 \mathrm{~h} 12$ \\
\hline Mustique-Bequia & 16 mai 2010 & 11,8 & $4 \mathrm{~h} 22$ \\
\hline $\begin{array}{c}\text { Bequia-Saint- } \\
\text { Vincent }\end{array}$ & 17 mai 2010 & 11,1 & $4 \mathrm{~h}$ \\
\hline $\begin{array}{c}\text { Kingston- } \\
\text { Chateaubelair } \\
\text { (Saint-Vincent) }\end{array}$ & 20 mai 2010 & 12,5 & $0 \mathrm{~h} 21$ \\
\hline $\begin{array}{c}\text { Baie des Pitons } \\
\text { (Sainte-Lucie) }\end{array}$ & 21 mai 2010 & 1,1 & $7 \mathrm{~h} 04$ \\
\hline $\begin{array}{c}\text { Sainte-Lucie- } \\
\text { Martinique }\end{array}$ & 22 mai 2010 & 24 & $31 \mathrm{~h} 37$ \\
\hline TOTAL & & 82,3 & \\
\hline
\end{tabular}

Fig. 13 - Données générales de l'expédition Grenade-Martinique, mai 2010.

Une dernière expérience a été menée le 10 mai 2011. Elle a pris, comme en 2008, la forme d'une simple navigation entre le nord de la Martinique et le sud de la Dominique. L'intérêt de cette expédition a été de faire varier un certain nombre de paramètres. Tout d'abord, elle a été réalisée dans la kanawa Loukouni Yabura d'une morphologie comparable à celle d'Akayouman mais longue de seulement $16 \mathrm{~m}$. De ce fait son équipage était limité à 24 personnes. De plus, nous avons aussi fait varier la composition de ce dernier par rapport aux précédentes expériences. Il était quasi exclusivement masculin et composé de jeunes militaires du $1^{\text {er }}$ RSMA $^{13}$ à qui nous avions fait subir un entraînement de plusieurs mois comparable à ceux préparant les autres expéditions. Malgré ces changements les données recueillies sont très proches de celles obtenues en mai 2008 et mai 2009 sur le même trajet. La distance de 23,7 MN a été ainsi parcourue en $6 \mathrm{~h} 37$.

Ainsi au cours de ces quatre expéditions, nous avons parcouru $270 \mathrm{MN}$ et traversé 12 canaux en 89 heures de navigation, le tout réparti sur 19 journées. Cela nous a permis de nous confronter à des conditions de mer très variées avec différentes compositions d'équipage. C'est donc une connaissance relativement

13. Régiment du service militaire adapté. 
précise que nous avons ainsi pu acquérir sur les techniques de navigation précolombiennes des Amérindiens antillais.

\section{Les données qualitatives}

Les expéditions que nous venons de décrire ont servi de support à une prise de données quantitatives précises. Mais au-delà, notre connaissance de la navigation en kanawa en termes qualitatifs repose maintenant sur huit années de pratique par deux groupes distincts (Karisko en Martinique et K'nawa en Guadeloupe), soit des centaines d'heures de navigations côtières et deux autres expéditions interinsulaires. L'association K'nawa a effectué le trajet entre la Dominique et Antigua en mai 2010 et les deux associations se sont retrouvées en mai 2013 pour une navigation avec deux embarcations entre la Martinique et la Guadeloupe. Cette expérience nous permet d'avancer sur une base relativement solide un premier ensemble d'éléments concernant les possibilités de navigation précolombiennes dans les Antilles.

La première question ayant un impact important est, en l'absence de balancier, le caractère instable des kanawa, confirmé tant par les sources historiques que par l'étude hydrostatique. Ce problème est en partie résolu par l'ajout d'un lest important (de l'ordre de $500 \mathrm{~kg}$ pour Akayouman). Cette spécificité des kanawa impose cependant un certain nombre de limitations. La principale d'entre-elles est que, lors de navigations sur une mer formée, seule une progression continue de l'embarcation permet d'assurer sa stabilité. En cas d'arrêt, la pirogue tend à se positionner travers à la lame, embarque de grandes quantités d'eau et chavire très rapidement. Cette nécessité de continuité dans la progression est une contrainte majeure pour les hypothèses impliquant des navigations de longue durée en haute mer.

Une fois cette première contrainte respectée, se pose la question des conditions de mer permettant la navigation en kanawa. Dans ce domaine plusieurs facteurs doivent être pris en compte. Il s'agit avant tout de la hauteur, de la fréquence et du sens de la houle. Concernant la hauteur de la houle, il nous est arrivé à quelques reprises de naviguer dans des mers présentant une houle dont la hauteur maximale était supérieure à $2 \mathrm{~m}$ pour une hauteur moyenne proche de $1,7 \mathrm{~m}^{14}$. Les kanawa naviguent étonnamment bien dans ce type de conditions sous réserve que la houle soit longue et régulière. Au regard de notre expérience, cela nous apparaît cependant comme des conditions limites pour une navigation présentant de bonnes conditions de sécurité. La navigation devient par contre beaucoup plus difficile lorsque la houle est courte voire croisée.

14. Les conditions maximales de navigation que nous ayons expérimentées à ce niveau correspondaient à une houle de 1,9 m de hauteur moyenne pour une hauteur maximale de $2,7 \mathrm{~m}$. Nos limites de sécurité ont été revues à la baisse suite à cette navigation mouvementée. 
Ensuite il nous est apparu que la principale difficulté était liée à la direction de la houle par rapport à la route suivie. Ainsi la meilleure allure correspond à une houle arrivant entre le 3/4 arrière et l'arrière de l'embarcation. C'est elle qui offre la meilleure vitesse associée à un relatif confort de navigation. Une houle venant entre le 3/4 avant et l'avant du bateau offre un confort de navigation relativement équivalent, mais il s'agit d'une allure plus lente. Enfin les plus mauvaises conditions de navigation correspondent à une houle arrivant par le travers du navire. Il n'est possible de tenir cette allure de façon confortable qu'avec une houle longue, régulière ou relativement faible sinon la kanawa court le risque de se remplir et de se renverser à chaque vague.

Comme nous l'avons dit, en haute mer, la progression de la kanawa doit être continue afin d'assurer sa stabilité. La limitation est donc liée à la capacité de l'équipage à maintenir cette progression. La plus longue navigation que nous ayons effectuée avec le même équipage a duré 14 heures. Nos données expérimentales montrent cependant clairement une baisse progressive de la vitesse au-delà de 6 à 7 heures de navigation. Cette donnée est évidemment fonction des capacités physiques et techniques de l'équipage. Quelles pourraient être les durées maximales de navigation efficace? Afin d'obtenir une évaluation nous nous sommes tournés vers les sports d'endurance desquels se rapproche en termes d'intensité la pratique de la navigation en kanawa. Ainsi le tour de France cycliste nous offre un premier exemple de la répétition d'efforts d'une durée moyenne de 5 heures durant trois semaines. Cela correspond en termes de durée au type de navigations d'île en île que nous avons réalisées lors de nos expéditions. Les meilleurs pratiquants d'ultratrail sont, quant à eux, capables de maintenir un effort efficace durant 20 à 30 heures ${ }^{15}$. La mise en place d'un système de rotation offrant la possibilité à une partie de l'équipage de se reposer à bord pendant de courtes périodes permet de prolonger la navigation. Il est cependant évident que cet allongement de la durée se fait au détriment de l'efficacité et que ce système atteint finalement ses limites du fait du peu de confort dont bénéficient les pagayeurs durant ces phases de repos.

Le dernier élément que nous souhaiterions évoquer est celui de la détermination du cap au cours de ces navigations. Bien qu'il y ait eu en permanence sur Akayouman un GPS, il avait pour unique fonction la prise de données et n'était pas utilisé pour déterminer la route à suivre. Il était en fait situé sur l'avant à plus de $17 \mathrm{~m}$ du barreur. Il y a une inter-visibilité continue possible entre les îles de l'archipel antillais uniquement interrompue au niveau du Passage d'Anegada situé entre Anguilla et les Îles Vierges (Torres et Rodriguez Ramos 2008; Ludlow 2011). Ainsi, au moment du départ, nous savions toujours vers où nous diriger. Cependant, une fois au niveau de la mer, cette visibilité se réduit

15. Il faut rappeler qu'il s'agit de sportifs de haut niveau et que ce type d'effort implique ensuite plusieurs semaines de récupération. Il s'agit donc d'une évaluation très haute (limite). 
de façon importante. Il en est de même du fait des variations des conditions atmosphériques. Ainsi, sous grain, la visibilité peut ne pas être supérieure à quelques dizaines de mètres. Cependant, une fois le cap connu, il peut être maintenu en cas de non-visibilité du point d'arrivée en prenant en compte la direction de la houle ou la position des différents objets célestes (les étoiles durant la nuit et dans une moindre mesure le soleil au cours de la journée). Ainsi la détermination de la route ne pose pas de problème majeur pour une navigation entre les îles de l'archipel.

\section{Les données quantitatives}

Des données quantitatives précises ont été recueillies lors de navigations effectuées le long des côtes de la Martinique en 2008 et $2009^{16}$ pour un total de $140 \mathrm{MN}$ ainsi que lors des quatre expéditions précédemment décrites. Les informations collectées correspondent donc à un total de $410 \mathrm{MN}$ de navigation. La procédure mise en place avait pour but de nous permettre de récolter trois types de données. Les premières concernaient la route suivie par la kanawa, sa vitesse durant le parcours (vitesse fond) ainsi que la durée totale de ce dernier (Figure 15). Cela a été réalisé grâce à la présence d'un GPS à bord d'Akayouman. Ensuite nous avons enregistré un certain nombre de données environnementales concernant la force et la direction du vent, la hauteur et la direction de la houle.

\begin{tabular}{|c|c|c|c|c|c|c|c|}
\hline Index & Horaire & Position & $\begin{array}{c}\text { Longueur } \\
\text { du segment } \\
(\mathrm{en} \mathbf{k m})\end{array}$ & \begin{tabular}{|c|}
$\begin{array}{c}\text { Longueur } \\
\text { du segment } \\
(\mathrm{en} \mathrm{MN})\end{array}$ \\
\end{tabular} & $\begin{array}{c}\text { Durée du } \\
\text { segment }\end{array}$ & $\begin{array}{c}\text { Vitesse du } \\
\text { segment } \\
\left(\text { en nd }^{\mathrm{a}}\right)\end{array}$ & $\begin{array}{l}\text { Vitesse du } \\
\text { segment } \\
(\mathrm{en} \mathrm{km} / \mathrm{h})\end{array}$ \\
\hline 167 & $\begin{array}{l}\text { 02-MAY-08 } \\
\text { 7:21:59AM }\end{array}$ & $\begin{array}{l}\text { N14 } 53.151 \\
\text { W61 } 08.631\end{array}$ & na & & na & na & na \\
\hline 168 & $\begin{array}{l}\text { 02-MAY-08 } \\
\text { 7:24:25AM }\end{array}$ & $\begin{array}{l}\text { N14 } 53.285 \\
\text { W61 } 08.677\end{array}$ & 0,26 & 0,14 & $2 \mathrm{~m} 26 \mathrm{~s}$ & 3,56 & 6,41 \\
\hline 169 & $\begin{array}{l}\text { 02-MAY-08 } \\
\text { 7:40:58AM }\end{array}$ & $\begin{array}{l}\text { N14 } 54.140 \\
\text { W61 } 09.042\end{array}$ & 1,71 & 0,93 & $16 \mathrm{~m} 33 \mathrm{~s}$ & 3,44 & 6,20 \\
\hline 170 & $\begin{array}{l}\text { 02-MAY-08 } \\
\text { 7:49:08AM }\end{array}$ & $\begin{array}{l}\text { N14 } 54.513 \\
\text { W61 } 09.239\end{array}$ & 0,78 & 0,42 & $8 \mathrm{~m} 10 \mathrm{~s}$ & 3,14 & 5,66 \\
\hline 171 & $\begin{array}{l}\text { 02-MAY-08 } \\
\text { 7:59:04AM }\end{array}$ & $\begin{array}{l}\text { N14 } 54.965 \\
\text { W61 } 09.454\end{array}$ & 0,92 & 0,50 & $9 \mathrm{~m} 56 \mathrm{~s}$ & 3,09 & 5,56 \\
\hline 172 & $\begin{array}{l}\text { 02-MAY-08 } \\
\text { 8:09:15AM }\end{array}$ & $\begin{array}{l}\text { N14 } 55.464 \\
\text { W61 } 09.638\end{array}$ & 0,98 & 0,53 & $10 \mathrm{~m} 11 \mathrm{~s}$ & 3,21 & 5,77 \\
\hline 173 & $\begin{array}{l}\text { 02-MAY-08 } \\
8: 24: 22 \mathrm{AM}\end{array}$ & $\begin{array}{l}\text { N14 } 56.255 \\
\text { W61 } 09.823\end{array}$ & 1,50 & 0,81 & $15 \mathrm{~m} 7 \mathrm{~s}$ & 3,31 & 5,95 \\
\hline 174 & $\begin{array}{l}\text { 02-MAY-08 } \\
\text { 8:34:19AM }\end{array}$ & $\begin{array}{l}\text { N14 } 56.763 \\
\text { W61 } 09.967\end{array}$ & 0,98 & 0,53 & $9 \mathrm{~m} 57 \mathrm{~s}$ & 3,25 & 5,85 \\
\hline
\end{tabular}

16. L'une d'entre elle a été réalisée de nuit, ce qui n'a pas posé de problèmes particuliers. 


\begin{tabular}{|c|c|c|c|c|c|c|c|}
\hline 175 & $\begin{array}{l}\text { 02-MAY-08 } \\
\text { 8:49:54AM }\end{array}$ & $\begin{array}{l}\text { N14 } 57.592 \\
\text { W61 } 10.241\end{array}$ & 1,61 & 0,87 & $15 \mathrm{~m} \mathrm{35s}$ & 3,49 & 6,28 \\
\hline 176 & \begin{tabular}{|l|} 
02-MAY-08 \\
8:54:59AM \\
\end{tabular} & $\begin{array}{l}\text { N14 } 57.884 \\
\text { W61 } 10.344 \\
\end{array}$ & 0,57 & 0,31 & $5 \mathrm{~m} 5 \mathrm{~s}$ & 3,74 & 6,73 \\
\hline 177 & \begin{tabular}{|l|} 
02-MAY-08 \\
9:11:36AM
\end{tabular} & $\begin{array}{l}\text { N14 } 58.815 \\
\text { W61 } 10.646 \\
\end{array}$ & 1,81 & 0,98 & $16 \mathrm{~m} 37 \mathrm{~s}$ & 3,63 & 6,54 \\
\hline 178 & \begin{tabular}{|l|} 
02-MAY-08 \\
9:24:09AM
\end{tabular} & $\begin{array}{l}\text { N14 } 59.549 \\
\text { W61 } 10.900 \\
\end{array}$ & 1,43 & 0,77 & $12 \mathrm{~m} 46 \mathrm{~s}$ & 3,73 & 6,72 \\
\hline 179 & $\begin{array}{l}\text { 02-MAY-08 } \\
\text { 9:37:10AM }\end{array}$ & $\begin{array}{l}\text { N15 } 00.235 \\
\text { W61 } 11.262 \\
\end{array}$ & 1,43 & 0,77 & $12 \mathrm{~m} 48 \mathrm{~s}$ & 3,72 & 6,70 \\
\hline 180 & \begin{tabular}{|l|} 
02-MAY-08 \\
9:46:25AM \\
\end{tabular} & $\begin{array}{l}\text { N15 } 00.757 \\
\text { W61 } 11.609 \\
\end{array}$ & 1,15 & 0,62 & $9 \mathrm{~m} 15 \mathrm{~s}$ & 4,14 & 7,46 \\
\hline 181 & \begin{tabular}{|l|} 
02-MAY-08 \\
9:54:57AM
\end{tabular} & $\begin{array}{l}\text { N15 } 01.231 \\
\text { W61 } 11.963 \\
\end{array}$ & 1,08 & 0,58 & $8 \mathrm{~m} 32 \mathrm{~s}$ & 4,22 & 7,59 \\
\hline 182 & \begin{tabular}{|c|} 
02-MAY-08 \\
10:02:02AM
\end{tabular} & $\begin{array}{l}\text { N15 } 01.594 \\
\text { W61 } 12.283 \\
\end{array}$ & 0,88 & 0,48 & $7 \mathrm{~m} 5 \mathrm{~s}$ & 4,14 & 7,45 \\
\hline 183 & \begin{tabular}{|c|} 
02-MAY-08 \\
10:12:59AM \\
\end{tabular} & $\begin{array}{l}\text { N15 } 02.105 \\
\text { W61 } 12.788 \\
\end{array}$ & 1,31 & 0,71 & $10 \mathrm{~m} 21 \mathrm{~s}$ & 4,22 & 7,59 \\
\hline 184 & $\begin{array}{c}\text { 02-MAY-08 } \\
\text { 10:23:31AM }\end{array}$ & $\begin{array}{ll}\text { N15 } & 02.584 \\
\text { W61 } 13.288 \\
\end{array}$ & 1,26 & 0,68 & $11 \mathrm{~m} 8 \mathrm{~s}$ & 3,77 & 6,79 \\
\hline 185 & \begin{tabular}{|c|} 
02-MAY-08 \\
10:35:28AM
\end{tabular} & $\begin{array}{l}\text { N15 } 03.165 \\
\text { W61 } 13.831 \\
\end{array}$ & 1,45 & 0,78 & $11 \mathrm{~m} 57 \mathrm{~s}$ & 4,04 & 7,28 \\
\hline 186 & \begin{tabular}{|c|} 
02-MAY-08 \\
10:44:44AM
\end{tabular} & $\begin{array}{l}\text { N15 } 03.602 \\
\text { W61 } 14.265\end{array}$ & 1,12 & 0,61 & $9 \mathrm{~m} 16 \mathrm{~s}$ & 4,03 & 7,25 \\
\hline 187 & $\begin{array}{c}\text { 02-MAY-08 } \\
\text { 10:54:37AM }\end{array}$ & $\begin{array}{l}\text { N15 } 04.089 \\
\text { W61 } 14.701 \\
\end{array}$ & 1,19 & 0,64 & $9 \mathrm{~m} 53 \mathrm{~s}$ & 3,99 & 7,19 \\
\hline 188 & $\begin{array}{c}\text { 02-MAY-08 } \\
\text { 11:10:44AM }\end{array}$ & $\begin{array}{l}\text { N15 } 04.885 \\
\text { W61 } 15.314 \\
\end{array}$ & 1,84 & 0,99 & $16 \mathrm{~m} 7 \mathrm{~s}$ & 3,81 & 6,85 \\
\hline 189 & \begin{tabular}{|c|} 
02-MAY-08 \\
11:27:48AM \\
\end{tabular} & $\begin{array}{l}\text { N15 } 05.714 \\
\text { W61 } 15.969 \\
\end{array}$ & 1,93 & 1,04 & $17 \mathrm{~m} 4 \mathrm{~s}$ & 3,77 & 6,79 \\
\hline 190 & \begin{tabular}{|c|} 
02-MAY-08 \\
11:38:40AM
\end{tabular} & $\begin{array}{l}\text { N15 } 06.281 \\
\text { W61 } 16.399 \\
\end{array}$ & 1,30 & 0,70 & $10 \mathrm{~m} 52 \mathrm{~s}$ & 3,99 & 7,18 \\
\hline 191 & $\begin{array}{c}\text { 02-MAY-08 } \\
\text { 11:48:12AM }\end{array}$ & $\begin{array}{l}\text { N15 } 06.768 \\
\text { W61 } 16.742 \\
\end{array}$ & 1,09 & 0,59 & $9 \mathrm{~m} 32 \mathrm{~s}$ & 3,81 & 6,86 \\
\hline 192 & \begin{tabular}{|l|} 
02-MAY-08 \\
11:54:18AM \\
\end{tabular} & $\begin{array}{l}\text { N15 } 07.074 \\
\text { W61 } 16.947 \\
\end{array}$ & 0,68 & 0,36 & $6 \mathrm{~m} 6 \mathrm{~s}$ & 3,72 & 6,69 \\
\hline 193 & $\begin{array}{l}\text { 02-MAY-08 } \\
\text { 12:05:04PM }\end{array}$ & $\begin{array}{l}\text { N15 } 07.549 \\
\text { W61 } 17.403 \\
\end{array}$ & 1,20 & 0,65 & $10 \mathrm{~m} 46 \mathrm{~s}$ & 3,72 & 6,69 \\
\hline 194 & $\begin{array}{l}\text { 02-MAY-08 } \\
\text { 12:14:44PM }\end{array}$ & $\begin{array}{l}\text { N15 } 07.979 \\
\text { W61 } 17.820 \\
\end{array}$ & 1,09 & 0,59 & $9 \mathrm{~m} 40 \mathrm{~s}$ & 3,76 & 6,77 \\
\hline 195 & \begin{tabular}{|l|} 
02-MAY-08 \\
12:22:22PM
\end{tabular} & $\begin{array}{l}\text { N15 } 08.345 \\
\text { W61 } 18.119 \\
\end{array}$ & 0,86 & 0,47 & $7 \mathrm{~m} 38 \mathrm{~s}$ & 3,76 & 6,76 \\
\hline 196 & $\begin{array}{l}\text { 02-MAY-08 } \\
\text { 12:32:59PM }\end{array}$ & $\begin{array}{l}\text { N15 } 08.805 \\
\text { W61 } 18.593\end{array}$ & 1,20 & 0,65 & $10 \mathrm{~m} 37 \mathrm{~s}$ & 3,77 & 6,78 \\
\hline
\end{tabular}


Approche expérimentale de la navigation précolombienne

\begin{tabular}{|c|c|c|c|c|c|c|c|}
\hline 197 & $\begin{array}{l}\text { 02-MAY-08 } \\
\text { 12:40:50PM }\end{array}$ & $\begin{array}{l}\text { N15 } 09.097 \\
\text { W61 } 18.948 \\
\end{array}$ & 0,83 & 0,45 & $7 \mathrm{~m} 51 \mathrm{~s}$ & 3,52 & 6,34 \\
\hline 198 & \begin{tabular}{|l|} 
02-MAY-08 \\
12:54:54PM
\end{tabular} & $\begin{array}{l}\text { N15 } 09.651 \\
\text { W61 } 19.622 \\
\end{array}$ & 1,58 & 0,86 & $14 \mathrm{~m} 4 \mathrm{~s}$ & 3,74 & 6,74 \\
\hline 199 & \begin{tabular}{|c|} 
02-MAY-08 \\
1:05:32PM
\end{tabular} & $\begin{array}{l}\text { N15 } 10.131 \\
\text { W61 } 20.164\end{array}$ & 1,32 & 0,71 & $10 \mathrm{~m} \mathrm{38s}$ & 4,14 & 7,45 \\
\hline 200 & \begin{tabular}{|c|} 
02-MAY-08 \\
1:10:33PM
\end{tabular} & $\begin{array}{l}\text { N15 } 10.369 \\
\text { W61 } 20.406\end{array}$ & 0,62 & 0,33 & $5 \mathrm{~m} \mathrm{1s}$ & 4,12 & 7,42 \\
\hline 201 & $\begin{array}{c}\text { 02-MAY-08 } \\
\text { 1:22:48PM }\end{array}$ & $\begin{array}{l}\text { N15 } 10.953 \\
\text { W61 } 20.947\end{array}$ & 1,45 & 0,78 & $12 \mathrm{~m} 15 \mathrm{~s}$ & 3,95 & 7,10 \\
\hline 202 & \begin{tabular}{|l|} 
02-MAY-08 \\
1:23:17PM \\
\end{tabular} & $\begin{array}{l}\text { N15 } 10.975 \\
\text { W61 } 20.965 \\
\end{array}$ & 0,05 & 0,03 & $29 \mathrm{~s}$ & 3,45 & 6,21 \\
\hline 203 & \begin{tabular}{|c|} 
02-MAY-08 \\
1:29:26PM
\end{tabular} & $\begin{array}{l}\text { N15 } 11.274 \\
\text { W61 } 21.226 \\
\end{array}$ & 0,72 & 0,39 & $6 \mathrm{~m} 9 \mathrm{~s}$ & 3,90 & 7,02 \\
\hline 204 & $\begin{array}{c}\text { 02-MAY-08 } \\
\text { 1:35:07PM }\end{array}$ & $\begin{array}{l}\text { N15 } 11.566 \\
\text { W61 } 21.451\end{array}$ & 0,67 & 0,36 & $5 \mathrm{~m} 41 \mathrm{~s}$ & 3,93 & 7,07 \\
\hline 205 & \begin{tabular}{|c|} 
02-MAY-08 \\
1:39:02PM
\end{tabular} & $\begin{array}{l}\text { N15 } 11.765 \\
\text { W61 } 21.596 \\
\end{array}$ & 0,45 & 0,24 & $3 \mathrm{~m} 55 \mathrm{~s}$ & 3,83 & 6,89 \\
\hline 206 & \begin{tabular}{|c|} 
02-MAY-08 \\
1:47:37PM
\end{tabular} & $\begin{array}{l}\text { N15 } 12.210 \\
\text { W61 } 21.921\end{array}$ & 1,01 & 0,54 & $8 \mathrm{~m} 35 \mathrm{~s}$ & 3,92 & 7,06 \\
\hline 207 & $\begin{array}{c}\text { 02-MAY-08 } \\
\text { 1:57:17PM }\end{array}$ & $\begin{array}{l}\text { N15 } 12.669 \\
\text { W61 } 22.357 \\
\end{array}$ & 1,15 & 0,62 & $9 \mathrm{~m} 40 \mathrm{~s}$ & 3,97 & 7,14 \\
\hline 208 & $\begin{array}{l}\text { 02-MAY-08 } \\
\text { 2:01:23PM }\end{array}$ & $\begin{array}{l}\text { N15 } 12.865 \\
\text { W61 } 22.484 \\
\end{array}$ & 0,43 & 0,23 & $4 \mathrm{~m} 6 \mathrm{~s}$ & 3,50 & 6,29 \\
\hline 209 & $\begin{array}{l}\text { 02-MAY-08 } \\
\text { 2:03:24PM }\end{array}$ & $\begin{array}{l}\text { N15 } 12.954 \\
\text { W61 } 22.456\end{array}$ & 0,17 & 0,09 & $2 \mathrm{~m} 1 \mathrm{~s}$ & 2,81 & 5,06 \\
\hline 210 & $\begin{array}{l}\text { 02-MAY-08 } \\
\text { 2:09:45PM }\end{array}$ & $\begin{array}{l}\text { N15 } 12.772 \\
\text { W61 } 22.152 \\
\end{array}$ & 0,64 & 0,35 & $6 \mathrm{~m} 21 \mathrm{~s}$ & 3,36 & 6,05 \\
\hline
\end{tabular}

Fig. 15 - Données GPS, traversée Martinique-Dominique, 2 mai 2008.

Mesure de dérive faite entre $9 \mathrm{~h}$ et $9 \mathrm{~h} 10: \mathrm{N} 0^{\circ} 0,116^{\prime} \mathrm{W}^{\circ} 0,162^{\prime}$; vitesse surface entre $9 \mathrm{~h}$ et $9 \mathrm{~h} 10: 3,03 \mathrm{nd}$; conditions très calmes, $50 \mathrm{~cm}$ de creux, vent faible.

Elles ont été complétées lors des expéditions par une mesure, effectuée régulièrement à l'aide d'un second GPS positionné sur un bateau suiveur, du phénomène de dérive lié aux effets conjoints du courant et du vent. Cette dernière donnée nous a ainsi permis d'évaluer l'impact des facteurs environnementaux et ainsi de déterminer la part réelle de l'équipage dans la progression de l'embarcation (calcul de la vitesse surface). Nous avons enfin enregistré la composition des équipages lors de ces différentes navigations. Ces données incluent la position sur la kanawa, le poids, l'âge et le sexe de chacun des équipiers. Elles prennent aussi en compte les éventuels changements effectués au sein de l'équipage en cours de navigation. 
Il nous est donc ainsi possible d'établir pour la première fois une estimation solide de la vitesse de croisière d'une kanawa. Lors des navigations d'entrâ̂nement effectuées sous le vent de la Martinique, les vitesses surfaces que nous avons calculées varient entre 3 et 3,5 nd. Au cours des différentes expéditions cette valeur a oscillé, à une exception près, entre 2,7 et 3,3 nd en fonction des conditions de navigation. Ainsi la seule mesure n'entrant pas dans cet intervalle a été effectuée dans des conditions de mer particulièrement difficiles dans plus de $2 \mathrm{~m}$ de creux avec un vent fort et sous grain. La vitesse surface d'Akayouman était alors de 2 nd. À l'inverse dans le cadre d'entrainements spécifiques et sur mer calme, il nous a été possible d'atteindre des vitesses proches de $5 \mathrm{nd}$. Cependant cette vitesse de pointe demande un effort intense à l'équipage qui n'a pu être maintenu plus de quelques minutes.

Ces mesures de vitesse nous permettent aussi de revenir sur la question de la capacité de transport d'une kanawa. Nous avons effectué nos différentes traversées avec un chargement d'environ $500 \mathrm{~kg}$ servant de lest ${ }^{17}$. Ainsi, avec une vitesse moyenne de 3 nd pour un équipage de 27 personnes et un chargement de $500 \mathrm{~kg}$, soit un déplacement de 4 t et un tirant d'eau de 0,41 m, on peut estimer la puissance développée par l'équipage à environ $1 / 2$ cheval (ch). Il est aussi intéressant de noter la faible influence du poids de la cargaison sur la vitesse de l'embarcation (Figure 16). Le principal facteur limitant la capacité d'emport des kanawa semble donc être le maintien d'une hauteur de francbord suffisante pour permettre une navigation confortable en limitant l'entrée de l'eau dans l'embarcation. Cette donnée varie évidemment en fonction des conditions de mer. Quoi qu'il en soit les kanawa sont aptes à transporter un équipage nombreux et une cargaison importante lors de navigations interinsulaires dans des mers formées.

\begin{tabular}{|c|c|c|c|c|}
\hline $\begin{array}{c}\text { Chargement } \\
(\mathbf{e n ~ t )}\end{array}$ & $\begin{array}{c}\text { Déplacement } \\
(\mathbf{e n ~ t )}\end{array}$ & $\begin{array}{c}\mathbf{S} \\
\left(\mathbf{e n ~}^{\mathbf{2}} \mathbf{)}\right.\end{array}$ & $\begin{array}{c}\text { Vitesse } \\
(\mathbf{e n} \mathbf{n d})\end{array}$ & $\begin{array}{c}\text { Perte de } \\
\text { vitesse (en \%) }\end{array}$ \\
\hline 0,5 & 4 & 0,323 & 3 & 0 \\
\hline 1 & 4,5 & 0,398 & 2,89 & $3,6 \%$ \\
\hline 1,5 & 5 & 0,812 & 2,8 & $6,7 \%$ \\
\hline 3 & 6,5 & 0,633 & 2,58 & $14,1 \%$ \\
\hline
\end{tabular}

Fig. 16 - Perte en vitesse en fonction de l'accroissement du déplacement (Billard et al. 2009).

17. Une expérience concluante de navigation en mer calme a aussi été menée avec près d'une tonne de chargement. 


\section{Bilan et perspectives}

Les cultures amérindiennes des Antilles font partie des rares exemples de civilisations basées sur la colonisation et l'occupation dans la longue durée d'un archipel océanique. Ainsi le cadre géographique pose d'entrée la question du rôle fondamental du fait maritime dans l'histoire précolombienne de la Caraïbe insulaire. Les recherches effectuées au sein d'un large corpus de textes européens de la période de contact (Bérard et al. 2016) nous ont tout d'abord permis d'approcher la nature du rapport entretenu par les Kalinago avec l'élément maritime; un rapport marqué par le développement d'une connaissance élaborée témoignant d'une véritable appropriation supportant sans doute un processus de territorialisation de cet espace trop souvent perçu comme homogène, dépourvu de lieu. Sans exclure une véritable conscience des risques, cette relation était aussi marquée par un esprit ludique de défi. Ces sources historiques, complétées par les quelques données archéologiques disponibles, nous ont aussi permis de dresser un panorama des moyens techniques dont disposaient les Amérindiens antillais dans le domaine de la navigation. Ils possédaient ainsi une flotte diversifiée apte à répondre à leurs différents besoins allant de la petite pêche côtière aux expéditions au long cours. Enfin et surtout, ces écrits nous ont permis de reconstituer la chaîne opératoire de construction des kanawa et ainsi de disposer d'une base solide pour le lancement de la partie expérimentale de notre projet.

Deux kanawa conformes à ces descriptions historiques ont ainsi été construites par un groupe d'Amérindiens Kali'ña de Guyane française à partir de leur savoir-faire traditionnel. Nous les avons utilisées pendant plusieurs années pour des navigations côtières comme pour des expéditions interinsulaires afin de récolter des données nous rendant aptes à effectuer une meilleure évaluation des capacités de navigation des populations précolombiennes des Antilles. Les kanawa sont des pirogues monoxyles à fargues pour lesquelles la navigation en haute mer ne présente pas de difficulté majeure. Cependant, leur morphologie ainsi que leur mode de propulsion, la pagaie, imposent un certain nombre de limitations. La première, qui est sans doute la principale, est la nécessité dans une mer formée de maintenir une progression permanente de l'embarcation afin d'assurer sa stabilité. Une autre donnée essentielle est la vitesse de croisière que nous avons pu estimer de façon raisonnable à 3 nd. La conjonction de ces deux éléments, au regard de la capacité d'un équipage à maintenir l'effort de pagayage, nous permet d'approcher une limite de la navigation en kanawa. La dernière limitation est liée aux conditions environnementales. Le facteur le plus contraignant à ce niveau est la houle. Cependant, il s'agit d'un système complexe intégrant la hauteur des vagues mais aussi leur fréquence et leur direction par rapport à la route. Certaines conditions nécessitent ainsi une modification du cap et donc un rallongement du trajet, d'autres s'opposent tout simplement à la navigation. Cependant l'observation 
des données météorologiques montre qu'il est possible de naviguer entre les îles des Antilles la majorité des jours de l'année.

Par rapport aux travaux antérieurs basés sur des calculs de dérive (Altes 2011; Callaghan 2001, 2011 et 2013), nos recherches nous permettent de passer de ce qui est possible en termes de navigation précolombienne à ce qui est le plus probable en réintégrant le facteur humain au travers de l'expérience pratique. Concernant la navigation entre les îles de l'archipel, les capacités de navigation des kanawa permettent d'envisager une fréquence élevée de voyages. De plus, à deux exceptions près, nous y reviendrons, chaque espace interinsulaire peut être traversé pendant la durée du jour. Enfin chaque trajet permet le transport de nombreux passagers et d'une importante cargaison. Cette capacité de navigation a non seulement permis de faire de l'archipel un véritable espace spécifique de civilisation (Hofman et Hoogland 2011; Hofman et van Duijvenbode 2011) mais a aussi pu servir de base au développement d'importants réseaux d'échanges (Knippenberg 2006) et à la constitution de micro-territoires interinsulaires (Bérard 2013a). Seules les traversées entre Tobago et Grenade et entre Anguilla et les Îles Vierges, $70 \mathrm{MN}$ environ pour chacune d'entre elles, imposent une navigation de nuit. Cependant une navigation dans le sens Tobago-Grenade bénéficiera globalement de courants favorables. Il en est de même pour un trajet dans le sens Anguilla-Virgin Gorda (Naval Oceanography Command Detachment 1986). Concernant la liaison entre Grenade et le Sud, malgré un allongement de la distance, les conditions environnementales sont plus en faveur d'un point d'atterrissage situé entre la Péninsule du Paria et l'île de Margarita, ce qui est conforme à différentes descriptions historiques de navigations réalisées par les Kalinago.

D'autres navigations directes plus longues entre le continent et l'archipel antillais ont été envisagées dans le cadre d'hypothèses migratoires ou de possibles réseaux échanges directs (Fitzpatrick 2013). Ainsi une origine mésoaméricaine du premier peuplement des Antilles a souvent été évoquée. Au regard de nos huit années d'expérience de la navigation en kanawa, l'existence de relations précolombiennes entre la Péninsule du Yucatan et l'ouest de Cuba, distants de $115 \mathrm{MN}$, nous semble rester dans le domaine du probable bien que la fréquence possible de ces échanges reste à évaluer. À l'inverse, si des traversées directes de la Mer des Caraïbes entre l'isthme colombien (ou un quelconque autre point du continent sud-américain) et les Grandes Antilles (au minimum $315 \mathrm{MN}$ ) sont peut-être réalisables, elles nous semblent bien peu probables. Les populations précolombiennes de l'archipel ne nous paraissent pas avoir été à même de les réaliser en toute sécurité et sur une base suffisamment régulière pour que les contacts correspondants aient pu jouer un rôle historique significatif. D'ailleurs, alors que nous envisageons de mettre sur pied une nouvelle expédition afin d'expérimenter des navigations directes plus longues que celles 
que nous avons réalisées jusqu'à présent, la réalisation d'un trajet de ce type en autonomie nous est apparue irréalisable et a été rapidement exclue.

Notre travail sur la navigation précolombienne dans les Antilles ne s'achève pas avec la publication de ces résultats. Il devrait se poursuivre à deux niveaux : par la mise en place d'une nouvelle expédition et par l'intégration des résultats de nos expérimentations à un modèle numérique. Concernant les projets d'expédition, trois hypothèses sont envisagées : une navigation entre Tobago et Grenade, la traversée du Passage d'Anegada entre Anguilla et les Îles Vierges ou une liaison entre le Yucatan et Cuba. La réalisation de ces projets dépendra de notre capacité à mettre en place les conditions matérielles et à réunir les importants moyens humains et financiers nécessaires à la réalisation de telles aventures. Une autre étape est d'ores et déjà en cours dans le cadre d'un travail doctoral intitulé Seascape Corridors conduit par E. Slayton à l'Université de Leiden ${ }^{18}$. L'objectif de ce travail est de construire un modèle numérique, basé sur un SIG, apte à effectuer une analyse de distance-coût ou à créer des lignes hypothétiques de trajet entre différents points côtiers en utilisant la mer comme surface de déplacement. Cette étude des déplacements au sein d'un paysage marin (seascape) sera basée sur le principe du chemin de moindre coût tel qu'il a été déjà été défini pour les paysages terrestres (Bell et Lock 2000). Plutôt que d'évaluer l'impact du relief sur les déplacements, l'analyse sera basée sur des variables telles que les courants ou les vents dominants. Ces variables seront traitées comme des équivalents à celles classiquement utilisées à terre comme des facteurs de frictions anisotropiques dans le cadre des analyses de distance-coût. Seul un nombre limité d'études (Altes 2011; Callaghan 2001 et 2011 ; Callaghan et Bray 2007) s'est jusqu'à présent intéressé à l'analyse de la navigation dans la Caraỉbe en termes de distance/coût. De plus ces travaux sont généralement centrés sur l'évaluation de possibles voyages de découverte ayant pu relier les différentes zones du continent à certaines îles de l'archipel antillais. Ainsi la question des trajets de retour n'y est souvent pas réellement traitée. Par ailleurs il est important de ne pas nous contenter d'évaluer des trajets possibles mais de mettre en relation ces routes avec des données environnementales, technologiques et anthropiques concrètes. L'intégration dans notre modèle de différentes couches de coût nous permettra de produire des évaluations de trajets plus réalistes. C'est à ce niveau que la prise en compte des données expérimentales comme la vitesse de croisière d'une kanawa, les conditions de mer navigables ou les capacités d'un équipage (puissance, durée d'effort) jouera un rôle fondamental. Enfin les données issues des nombreuses

18. Cette recherche fait parti du projet « Island networks: modelling inter-community social relationships in the Lesser Antilles across the historical divide (AD 1000-1800)» dirigé par le professeur Corinne L. Hofman et financé par l'organisation néerlandaise pour la recherche scientifique (NWO), projet no 360-62-060. 
navigations réellement effectuées par Akayouman nous permettront de tester de façon directe la validité des différents trajets théoriques produits par le modèle numérique. Ainsi il nous est aujourd'hui possible de comparer nos données avec celles obtenues par Richard Callaghan concernant la traversée du canal entre la Guadeloupe et Antigua (Callaghan 2010). Il faut cependant noter que le type de pirogue basé sur le canot Stargate (Bérard et al. 2016) qu'il a utilisé pour sa simulation est très différent du nôtre. Par contre les données concernant la vitesse utilisée (vitesse entre 2 et 3,4 nd) sont très comparables à celles que nous avons obtenues expérimentalement. Nous avons effectué la traversée dans le sens sud-nord en $11 \mathrm{~h} 35$ dans une mer formée sans rencontrer de difficulté majeure. Nous ne pouvons donc que partager les conclusions de Richard Callaghan "Given the results, the reasons for the paucity of sites (archaic) south of the passage are unlikely to be related to oceanographic or technological deficiencies 》(Callaghan 2011, p. 137). Ne disposant pas des données précises issues de la simulation, il nous est pour l'instant difficile de pousser plus loin la comparaison. Nous voyons cependant comment va pouvoir maintenant s'instaurer un dialogue entre différents types d'approches. Nous espérons ainsi que notre travail permettra d'affiner notre compréhension des capacités de navigation des populations précolombiennes antillaises et nous rendra à même d'apporter des réponses plus précises aux différentes questions dont le traitement par l'archéologie maritime préhistorique ne pouvait être jusqu'à présent que théorique. *

* Manuscrit reçu en décembre 2014, accepté pour publication en mai 2016.

Remerciements - Richard Callaghan nous a aimablement transmis les documents relatifs aux études climatiques menées par l'U.S. Navy. Qu'il soit ici remercié. Ce travail ne doit son existence qu'à l'association Karisko, sa direction, ses ouboutou, tous ses membres et ses partenaires. Rien n'aurait été possible sans leur passion, leur dynamisme, leur douce et furieuse folie. Que soient remerciés par ordre d'apparition: l'association T'lewuyu, nos frères de terre ferme, créateurs de kanawa, que Tamouchi veille sur eux; ceux qui ensuite ont entretenu avec « amour» Akayouman durant ces années; les Cannibales de Martinique et d'ailleurs, les Carib Sea Warriors de la Dominique et le $1^{\text {er }}$ RSMA de Martinique qui ont constitué les équipages des différentes expéditions, yo pa té janmè kayé; nos coachs qui ont préparé ces équipages, ceux qui les ont accompagnés et filmés, les ont nourris et ont assuré leur sécurité et leur confort; tous nos partenaires qui de la Grenade à Antigua nous ont accueillis, logés et bien souvent nourris lors de notre passage, ils sont ces Caraïbes d'une autre île mais d'une même nation. 
Approche expérimentale de la navigation précolombienne

\section{Références citées}

Altes Christopher

2011, « A brief note on currents, current archaeologists, and ancient fire-tempered pots », The Florida anthropologist, 64 (2), p. 113-118.

BELL Tyler et Gary Lock

2000, « Topographic and cultural influences on walking the Ridgeway in later Prehistoric times », in Gary Lock (éd.), Beyond the map, archaeology and spatial technologies, IOS Press, Amsterdam, p. 85-100.

BENNET Jenny (éd.)

2009, Sailing into the past. Learning from replica Ships, Seaforth Publishing, Barnsley. BÉRARD Benoît

2004, Les premières occupations agricoles de l'arc antillais, migrations et insularité, Archaeopress (BAR, International series, 1299; Paris monographs in American archaeology, 15), Oxford.

2007, « Le phénomène pionnier agro-céramiste antillais : vers une vision archipélique », Les nouvelles de l'archéologie, 108-109, p. 70-77.

2008, « La mission archéologique française en Dominique », Les nouvelles de l'archéologie, 111-112, p. 95-100.

2013a, « Penser les territoires de l'histoire amérindienne des Antilles », Outre-Mers, 378-379, p. 151-164.

2013b, « L'occupation saladoïde ancienne de la Dominique, vers une nouvelle définition des territoires culturels précolombiens "), in Benoît Bérard (dir.), Martinique, terre amérindienne. Une approche pluridisciplinaire, Sidestones Press, Leiden, p. 235-246.

Bérard Benoît, Jean-Yves Billard, Thierry L'Etang, Guillaume Lalubie, Constantino Nicolizas, Bruno Ramstein et Emma Slayton

2016, «Technologie du fait maritime chez les Kalinago des Petites Antilles au XVI ${ }^{\mathrm{e}}$ et $\mathrm{XVII}^{\mathrm{e}}$ siècles », Journal de la société des américanistes, 102 (1), p. 129-158.

Billard Jean-Yves, Benoît Bérard et Bruno Ramstein

2009, « Apports de l'hydrostatique à l'archéologie expérimentale. Étude d'une pirogue de haute mer (kanawa) », $19^{e}$ Congrès français de mécanique [en ligne] (Marseille, 24 au 28 août 2009), http://documents.irevues.inist.fr/bitstream/ handle/2042/36639/772.pdf?sequence=1, consulté sur I-Revues le 09/12/16.

Booy Theodoor de

1913, «Lucayan artifacts from the Bahamas », American anthropologist, 15, p. 1-7.

BRETON Raymond

1665, Dictionnaire caraïbe-françois meslé de quantité de Remarques historiques pour l'esclaircissement de la Langue, Gilles Bouquet, Auxerre.

Callaghan Richard

2001, «Ceramic age seafaring and interaction potential in the Antilles. A computer simulation », Current anthropology, 42 (2), p. 11-22.

2010, «Crossing the Guadeloupe passage in the archaic age », in Scott Fitzpatrick et Ann Ross (éd.), Island shores, distant pasts, University Press of Florida, Gainesville, p. 127-147. 
Callaghan Richard

2011, « Patterns of contact between the islands of the Caribbean and the surrounding mainland as a navigation problem », in Curet L. Antonio et Mark W. Hauser (éd.), Islands at the crossroads: migration, seafaring, and interaction in the Caribbean, University of Alabama Press, Tuscaloosa, p. 59-72.

2013, " Archaeological views of seafaring in the Caribbean », in William F. Keegan, Corinne L. Hofman et Reniel Rodríguez Ramos (éd.), The Oxford handbook of Caribbean archaeology, Oxford University Press (Oxford handbooks), Oxford, p. 283-295.

Callaghan Richard et William Bray

2007, « Simulating prehistoric sea contacts between Costa Rica and Colombia », Journal of Island and Coastal archaeology, 2, p. 4-23.

Capelotti Peter Joseph

2012, "The theory of the archaeological raft: motivation, method, and madness in experimental archaeology », Experimental Archaeology [en ligne], 2012 (1), http://exarc.net/issue-2012-1/ea/theory-archaeological-raft-motivation-methodand-madness-experimental-archaeology, consulté le 08/12/16.

Chalifoux Jean-Jacques

1998, « Chamanisme et couvade chez les Galibi de la Guyane française », Anthropologie et sociétés, 22 (2), p. 99-123.

Christensen Arne Emil et Ian Morisson

1976, « Experimental archaeology and boats », International journal of nautical archaeology and underwater exploration, 5 (4), p. 275-284.

Coates John, Sean McGrail, David Brown, Edwin Gifford, Gerald Grainge, Basil Greenhill, Peter Marsden, Boris Rankov, Colin Tipping et Edward Wright

1995, "Experimental boat and ship archaeology: principles and methods ", The International journal of nautical archaeology, 24 (4), p. 293-301.

COLleCtiF KarisKo

2011, « La renaissance des kanawas », Chasse marée, 230, p. 32-39.

Crumlin-Petersen Ole

1995, « Experimental archaeology and ships - bridging the arts and the sciences », The International journal of nautical archaeology, 24 (4), p. 303-306.

Crumlin-Petersen Ole et Sean McGrail

2006, "Some principles for the reconstruction of ancient boat structures », The International journal of nautical archaeology, 35 (1), p. 53-57.

Crumlin-Pedersen Ole et Max Vinner (éd.)

1986, Sailing into the past. Proceedings of the International seminar on replicas of ancient and medieval vessels, Viking Ship Museum, Roskilde, 1984.

Di Méo Guy

1998, Géographie sociale et territoires, Nathan, Paris, 1998.

FitZPATRICK Scott M.

2013, " Seafaring capacities in the Pre-Columbian Caribbean », Journal of maritime archaeology, 8 (1), p. 101-138.

François Sophie

2003, « Les pirogues du Maroni », In situ, 3, p. 66-82. 
Approche expérimentale de la navigation précolombienne

Hofman Corinne L. et Ann van Duijvenbode (éd.)

2011, Communities in contact. Essays in archaeology, ethnohistory et ethnography of the Amerindian circum-Caribbean, Sidestone Press, Leiden.

Hofman Corine L. et Menno Hoogland

2011, « Unravelling the multi-scale networks of mobility and exchange in the pre-colonial circum-Caribbean », in Corine L. Hofman et Ann van Duijvenbode (éd.), Communities in contact. Essays in archaeology, ethnohistory et ethnography of the Amerindian circum-Caribbean, Sidestone Press, Leiden, p. 15-43.

KNIPPENBERG Sebastiann

2006, Stone artefact production and exchange among the Northern Lesser Antilles, Ph.D. dissertation, Faculty of Archaeology, Leiden University, Leiden.

LABAT Jean-Baptiste

1742 [1694-1705], Nouveau voyage aux Isles de l'Amérique, contenant l'histoire naturelle de ces pays, l'origine, les mours, la religion et le gouvernement des habitans anciens \& modernes..., Charles-Jean-Baptiste Delespine, Paris, 8 vol.

LudLow Mark M.

2011, « For the earliest Pre-Columbian human migrants into the Lesser Antilles of the Caribbean, Grenada was visible from either Trinidad or Tobago. A barrier only by incomplete arithmetic calculation », in Samantha Rebrovich (éd.), Proceedings of the XXIII Congress of the International Association for Caribbean Archaeology (Antigua, 29 June-3 July 2009), Dockyard Museum, Antigua, p. 607-614.

McGrail Sean

1974, « Part 1. Building the replica », in McGrail Sean et McKee Eric, The building and trials of the replica of an ancient boat: the Gokstad faering, National Maritime Museum (Maritime monographs and reports, 11), London.

2009, « Experimental archaeology: replicas and reconstructions », in Jenny Bennet (éd.), Sailing into the past. Learning from replica ships, Seaforth Publishing, Barnsley, p. 16-23.

McKusick Marshall B.

1960, Aboriginal canoes in the West Indies, Dept. of Anthropology, Yale University (Yale University publications in anthropology, 63), New Haven.

Naval Oceanography Command Detachment

1986, U.S. Navy climatic study of the Caribbean Sea and Gulf of Mexico. Vol. 2. East Caribbean Sea, Naval Oceanography Command, Asheville (NC).

Nicholson Desmond A.

1976, « Pre-Columbian seafaring capabilities in the Lesser Antilles », in Jacques Petitjean Roget et Ripley P. Bullen (ed), Proceedings of the sixth International congress for the study of pre-Columbian cultures of the Lesser Antilles [Pointe à Pitre, Guadeloupe, July 6-12, 1975], Florida State Museum/University of Florida, Gainesville, p. 98-105.

NúÑEZ JiMÉNEZ Antonio

1992, Remando del Amazonas al Caribe, Lunwerg Editores S.A., Barcelona.

1994, En canoa, por el mar de las Antillas, Patronato de la ciudad colonial de Santo Domingo (Quinto Centenario, Serie Estudios, 11), Santo Domingo 
Benoît BÉrARD et al.

Ostapkowicz Joanna, Christopher B. Ramsey, Fiona Brock, Tom Higham, Alex C. Wiedenhoeft, Erika Ribechini, Jeannette J. LuCEJKo et Samuel WiLson

2012, « Chronologies in wood and resin: AMS 14C dating of pre-Hispanic Caribbean wood sculpture », Journal of archaeological science, 39, p. 2238-2251.

Robiou Lamarche Sebastian

2009, Caribes creencias y rituales, Punto y Coma, San Juan.

TORres Josua et Reniel Rodriguez RAmos

2008, « The Caribbean. A continent divided by water », in Basil A. Reid (éd.), Archaeology and geoinformatics. Case studies from the Caribbean, Tuscaloosa, University of Alabama Press, p. 13-29. 